\title{
ON THE SCHWARZ ALGORITHMS FOR THE ELLIPTIC EXTERIOR BOUNDARY VALUE PROBLEMS
}

\author{
Faker Ben Belgacem ${ }^{1}$, Michel Fournié ${ }^{1}$, Nabil Gmati ${ }^{2}$ and Faten Jelassi ${ }^{3}$
}

\begin{abstract}
Tuning the alternating Schwarz method to the exterior problems is the subject of this paper. We present the original algorithm and we propose a modification of it, so that the solution of the subproblem involving the condition at infinity has an explicit integral representation formulas while the solution of the other subproblem, set in a bounded domain, is approximated by classical variational methods. We investigate many of the advantages of the new Schwarz approach: a geometrical convergence rate, an easy implementation, a substantial economy in computational costs and a satisfactory accuracy in the numerical results as well as their agreement with the theoretical statements.
\end{abstract}

Mathematics Subject Classification. 35J20, 65N38, 65N55.

Received: April 15, 2004. Revised: March 29, 2005.

\section{INTRODUCTION AND NOTATIONS}

Formulating the integral equations, which are relevant for the exterior value problems and commonly used in scientific computing, requires an explicit knowledge of the elementary solution - also called the Green function of the model we deal with (see $[5,8,10,16,17,29,36,37,46]$ ). The point is that these Green functions are not usually accessible at reasonable costs. Apart from some problems such as, e.g., the Poisson, Helmholtz, Schrödinger, Maxwell, elastostatic and plates problems, an efficient calculation of these Green kernels is likely to fail for most differential equations with non-constant coefficients. An alternative solution, to circumvent these limitations, is to resort to coupling methods between the finite element method and the boundary element method (FEM/BEM), proposed in [42] (see also [47]) and theoretically studied by Johnson and Nédélec in [25]. It is a hopeful tool to extend the application of the integral equations to a larger class of partial differential equations.

Keywords and phrases. Boundary integral equations, boundary element methods, finite element methods, coupling methods, domain decomposition techniques, Schwarz algorithm.

1 MIP (UMR CNRS 5640), UPS, 118 route de Narbonne, 31062 Toulouse, France. belgacem@mip.ups-tlse.fr;

fournie@mip.ups-tlse.fr

2 LAMSIN, IPEIN, Campus Universitaire, Route Mrazka, 8000 Nabeul, Tunisia. nabil.gmati@ipein.rnu.tn

3 LAMSIN, FSB, Jarzouna, 7021 Bizerte, Tunisia. faten.jelassi@lamsin.rnu.tn

This work was supported by the Ministère de la Recherche Scientifique, de la Technologie et du Développement des Compétences (MRSTDC, TUNISIA) under the LAB-STI-02 program and the Coopération avec les Chercheurs Tunisiens Résidents à l'Étranger program (2004).

(c) EDP Sciences, SMAI 2005 
The coupling approach consists in truncating the computational domain by the introduction of a fictitious boundary, on which an artificial condition is derived via a non-local singular integral representation. Many variational formulations are already applied to the reduced problem. One of them is discussed in [25] and its good behavior illustrated by some numerical experiments (see also [42,47]). Later work by Jami and Lenoir (see $[20-22,40,41])$ brings about further improvements to the (FEM/BEM) methodology by suitably considering an overlapping domain decomposition technique, so as to write down more general formulations of the artificial condition. The representation of this non-standard condition is based on regular kernels (versus singular kernels for the Johnson-Nédélec coupling method); the main effect of it is to avoid the special treatment due to the singularities of the Green function and to improve the conditioning of the stiffness matrix and thus of the system to be inverted.

The advances achieved in the mathematical ground for the exterior problems arise much interest on the related practical issues such as finding out performing algorithms in order to solve the discrete problem is the crucial point. Recall that, for symmetric boundary value problems, the counterpart of the coupling approximation breaks the symmetry and substantially alters the stiffness matrix sparsity (see [34]). A large number of the solvers, coming from the tremendous progress in the domain decomposition methods (see $[30,31,38,43]$ ), can be explored and possibly applied to problems set on unbounded domains. Currently, not much work achieved on the Schwarz algorithm for exterior problems has provided encouraging results (see, for instance, $[2,9,32,33]$ ). The core of this contribution is the new iterative method introduced in [3], for the Poisson model set in open domains, for which a full analysis is detailed. Naturally, the new algorithm has been filed of the Schwarz methods (see [39]), since it is an adaptation of the original (Schwarz) procedure to the unboundedness of the domain which allows to reduce the computational costs, owing to the integral representations. During the iterations, we modify one of the subproblems, the one where the condition at infinity is taken into account. This enables us to give an explicit expression of its solution. The other subproblem is set in the bounded sub-domain, for which a local Dirichlet or Neumann condition results from the previous step solution; it is solved by Lagrangian finite element methods. The iterative process is stopped when a given tolerance is obtained, and the computed solution is considered to be a consistent approximation of the exact model.

The outline of the paper ${ }^{1}$ is as follows. The variational framework of [30] is extended to the unbounded domains. Afterward, it is worked out to fit our modified Schwarz method. Then, applying the Cauchy fixed point theory, we exhibit a geometrical convergence of it (the new Schwarz procedure). Reformulating it allows to underline the possible connections with different (FEM/BEM) methods. Finally, analytical calculations for separable geometries together with some numerical computations are reported to highlight the reliability of the new Schwarz algorithm.

Some functional notations - Let $\Omega$ be a bounded domain in $\mathbb{R}^{d}, d=2,3$, with a Lipschitz boundary $\Gamma$. The Lebesgue space $L^{2}(\Omega)$ of square integrable functions is endowed with the natural norm $\|\cdot\|_{L^{2}(\Omega)}$ and we set $\mathrm{L}^{2}(\Omega)=L^{2}(\Omega)^{d}$. We need also some Sobolev spaces, $H^{1}(\Omega)$ involves all the functions that are in $L^{2}(\Omega)$ as well as their partial derivatives. The set of the traces over $\Gamma$ of all the functions of $H^{1}(\Omega)$ is denoted $H^{\frac{1}{2}}(\Gamma)$ and $H^{-\frac{1}{2}}(\Gamma)$ is its dual (see $\left.[1,10]\right)$. All along this work, for any $\Gamma$ which separates an external and an internal regions $\Omega^{e}$ and $\Omega^{i}$, the symbol [.] stands for the jump across $\Gamma$, i.e. $[\varphi]=\varphi_{\left.\right|_{\Gamma}}^{e}-\varphi_{\left.\right|_{\Gamma}}^{i}$ for any function $\varphi=\left(\varphi^{i}=\varphi_{\left.\right|_{\Omega^{i}}}, \varphi^{e}=\varphi_{\left.\right|_{\Omega^{e}}}\right)$.

\footnotetext{
1 In many places of the manuscript, we refer the reader to the Ph.D. Thesis [24] of the fourth author, in preparation. We do want to stress that the commonly accepted rule, that is, such a referencing is tolerated only for non-essential details and for some more extensions, is fully respected.
} 


\section{The Laplace-Neumann PROBlem in $\mathbb{R}^{3}$}

Let $\Gamma$ be a bounded and closed surface in $\mathbb{R}^{3}$, delimiting the internal and the external domains $\Omega_{\Gamma}^{i}$ and $\Omega_{\Gamma}^{e}$. We assume that it is smooth and we denote by $\boldsymbol{n}$ the unit normal (to $\Gamma$ ) oriented from $\Omega_{\Gamma}^{e}$ toward $\Omega_{\Gamma}^{i}$. For a given data $g \in H^{-\frac{1}{2}}(\Gamma)$, the Laplace-Neumann problem consists in: finding $u$ such that

$$
\begin{aligned}
-\Delta u & =0, & & \text { in } \Omega_{\Gamma}^{e}, \\
\partial_{\boldsymbol{n}} u & =g, & & \text { on } \Gamma, \\
\nabla u & =\left(\frac{1}{|\boldsymbol{x}|}\right), & & \text { at infinity. }
\end{aligned}
$$

Due to the unboundedness of the domain $\Omega_{\Gamma}^{e}$, the variational formulation is based on the weighted Sobolev space (also known as the Beppo-Levi space) defined by

$$
W^{1}\left(\Omega_{\Gamma}^{e}\right)=\left\{v ; \frac{v}{\sqrt{1+|\boldsymbol{x}|^{2}}} \in L^{2}\left(\Omega_{\Gamma}^{e}\right), \quad \nabla v \in \mathrm{L}^{2}\left(\Omega_{\Gamma}^{e}\right)\right\} .
$$

We refer to [10] for some of its properties. Expressing the problem (1)-(3) in a weak form leads to: find $u \in W^{1}\left(\Omega_{\Gamma}^{e}\right)$ such that

$$
\int_{\Omega_{\Gamma}^{e}} \nabla u \nabla v \mathrm{~d} \boldsymbol{x}=\int_{\Gamma} g v \mathrm{~d} \gamma, \quad \forall v \in W^{1}\left(\Omega_{\Gamma}^{e}\right) .
$$

This problem has a unique solution. When an approximation of the problem is aimed, (4) can not be used for scientific computing. So, a current numerical approach is to resort to the integral equations (see $[10,36])$. They are based on a suitable representation of $u$, picked from the potential theory (see [8])

$$
u(\boldsymbol{x})=\int_{\Gamma} u_{\mid \Gamma}(\cdot) \partial_{\boldsymbol{n}} G(\boldsymbol{x}-\cdot) \mathrm{d} \gamma-\int_{\Gamma} g(\cdot) G(\boldsymbol{x}-\cdot) \mathrm{d} \gamma, \quad \forall \boldsymbol{x} \in \Omega_{\Gamma}^{e},
$$

where $G(\boldsymbol{x})=\frac{1}{4 \pi|\boldsymbol{x}|}$ is the Green function.

Remark 2.1. The integral representation (5) needs to be adapted to $\Gamma$. To provide the general form of it, we assume that $\Gamma$ is a Lipschitzian boundary tolerating corners. Let $\boldsymbol{x}$ be on $\Gamma$ and denote $\theta$ the measure of the external angle made by $\Gamma$ around $\boldsymbol{x}$ as shown in Figure 1. Then, identity (5) becomes (see [36])

$$
\frac{\theta}{2 \pi} u(\boldsymbol{x})=\int_{\Gamma} u_{\mid \Gamma}(\cdot) \partial_{\boldsymbol{n}} G(\boldsymbol{x}-\cdot) \mathrm{d} \gamma-\int_{\Gamma} g(\cdot) G(\boldsymbol{x}-\cdot) \mathrm{d} \gamma .
$$

At the vicinity of the regular points, the boundary is flat and thus $\theta=\pi$. We do not address this situation in the sequel, and we only consider a smooth boundary $\Gamma$ (i.e. with no angular points).

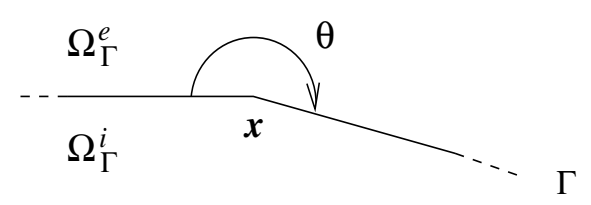

Figure 1. Example of an angular boundary of the obstacle $\Gamma$. 
Notice that (5) permits an easy reconstruction of the solution $u$, on the whole domain $\Omega_{\Gamma}^{e}$, from the knowledge of $u_{\mid \Gamma}=\varphi$. This function $\varphi$ is computed by solving the following integral equation

$$
\frac{\varphi}{2}(\boldsymbol{x})-\int_{\Gamma} \varphi(\cdot) \partial_{\boldsymbol{n}} G(\boldsymbol{x}-\cdot) \mathrm{d} \gamma=-\int_{\Gamma} g(\cdot) G(\boldsymbol{x}-\cdot) \mathrm{d} \gamma, \quad \forall \boldsymbol{x} \in \Gamma .
$$

The coerciveness and the symmetry of problem (6) are readily checked. The variational discretization of it by the boundary finite elements so as the derivation of the associated algebraic system are detailed in $[5,7,8,10,16-18,36,37,45]$. We recall two negative consequences of this type of discretization

$i$. The computation of the stiffness matrix entries requires a particular treatment of the singular kernels in the integral equation.

ii. This (stiffness) matrix is full with a condition number growing like the inverse of the mesh size. It is not easy to solve efficiently the problem even thought performing preconditioners (see $[7,45]$ ) were recently introduced.

An alternative to overcome such complexities consists in truncating the unbounded domain $\Omega_{\Gamma}^{e}$ by introducing a fictitious boundary $\Sigma$ not intersecting with $\Gamma$. Then, we consider the Laplace equation (1) set in $\Omega_{\Sigma}^{c}$, the annular domain delimited by $\Gamma$ and $\Sigma$ with the Neumann condition (2) on $\Gamma$, and an artificial condition on $\Sigma$ obtained from formula (5). For commodity, this last condition is rewritten, using the notations $V_{\Gamma}$ and $K_{\Gamma}$ for the simple and the double layer potentials,

$$
u=K_{\Gamma}\left(u_{\mid \Gamma}\right)-V_{\Gamma}(g), \quad \text { on } \Sigma .
$$

It is a non-local condition and generates a coupling between $u_{\mid \Sigma}$ and $u_{\mid \Gamma}$; it can be viewed as an absorbing condition of a non-standard Dirichlet type. To take it into account in a variational form, we denote by $\mathcal{R}_{\Sigma}$ an arbitrary stable extension of traces on $\Sigma$ that vanishes on $\Gamma$, and we decompose the solution $u$ into the sum $\mathcal{R}_{\Sigma}\left(K_{\Gamma}\left(u_{\mid \Gamma}\right)-V_{\Gamma} g\right)+\underline{u}$. The new unknown $\underline{u}$ has the same trace on $\Gamma$ as $u\left(i . e . \underline{u}_{\mid \Gamma}=u_{\mid \Gamma}\right)$ and belongs to the standard Sobolev space

$$
H_{0, \Sigma}^{1}\left(\Omega_{\Sigma}^{c}\right)=\left\{v \in H^{1}\left(\Omega_{\Sigma}^{c}\right) ; \quad v_{\mid \Sigma}=0\right\} .
$$

The (truncated) variational problem can then be formulated as follows: find $\underline{u} \in H_{0, \Sigma}^{1}\left(\Omega_{\Sigma}^{c}\right)$ such that

$$
\begin{aligned}
\int_{\Omega_{\Sigma}^{c}} \nabla \underline{u} \nabla v \mathrm{~d} \boldsymbol{x}+\int_{\Omega_{\Sigma}^{c}} \nabla \mathcal{R}_{\Sigma}\left(K_{\Gamma}\left(\underline{u}_{\mid \Gamma}\right)\right) \nabla v \mathrm{~d} \boldsymbol{x}= \\
\int_{\Gamma} g v \mathrm{~d} \gamma+\int_{\Omega_{\Sigma}^{c}} \nabla \mathcal{R}_{\Sigma}\left(V_{\Gamma} g\right) \nabla v \mathrm{~d} \boldsymbol{x}, \quad \forall v \in H_{0, \Sigma}^{1}\left(\Omega_{\Sigma}^{c}\right) .
\end{aligned}
$$

The coupling of finite elements method and boundary elements method, denoted henceforth by (FEM/BEM), consists in the approximation of problem (8) by the Lagrangian finite elements. The resulting stiffness matrix suffers from the non-symmetry and is also partially full due to a (full) block related to the degrees of freedom located on $\Sigma$ and $\Gamma$. The (FEM/BEM)-approach is studied in many publications (see, e.g., $[22,26]$ ) and is implemented in the computing code MELINA used for our computations (see [34]).

Remark 2.2 (General coupling procedures). The artificial boundary condition (7) may be written using an intermediary boundary surface $\Sigma_{*}$ immersed in the domain $\Omega_{\Sigma}^{c}$, instead of $\Gamma$ ( $\Sigma_{*}$ has a simple shape). In this case, it is transformed into

$$
u=K_{\Sigma_{*}}\left(u_{\mid \Sigma_{*}}\right)-V_{\Sigma_{*}}\left(\partial_{\boldsymbol{n}} u_{\mid \Sigma_{*}}\right), \quad \text { on } \Sigma .
$$

When $\Sigma_{*} \cap \Sigma=\emptyset$, we obtain the Jami-Lenoir method (see [22]) while the limit case $\Sigma_{*}=\Sigma$ gives the JohnsonNédélec procedure (see [25]). Then, the boundary condition is expressed by

$$
u=\left(\frac{1}{2} I_{\Sigma}+K_{\Sigma}\right)\left(u_{\mid \Sigma}\right)-V_{\Sigma}\left(\partial_{\boldsymbol{n}} u_{\mid \Sigma}\right), \quad \text { on } \Sigma,
$$

where the symbol $I_{\Sigma}$ denotes the identity operator on $H^{\frac{1}{2}}(\Sigma)$. 


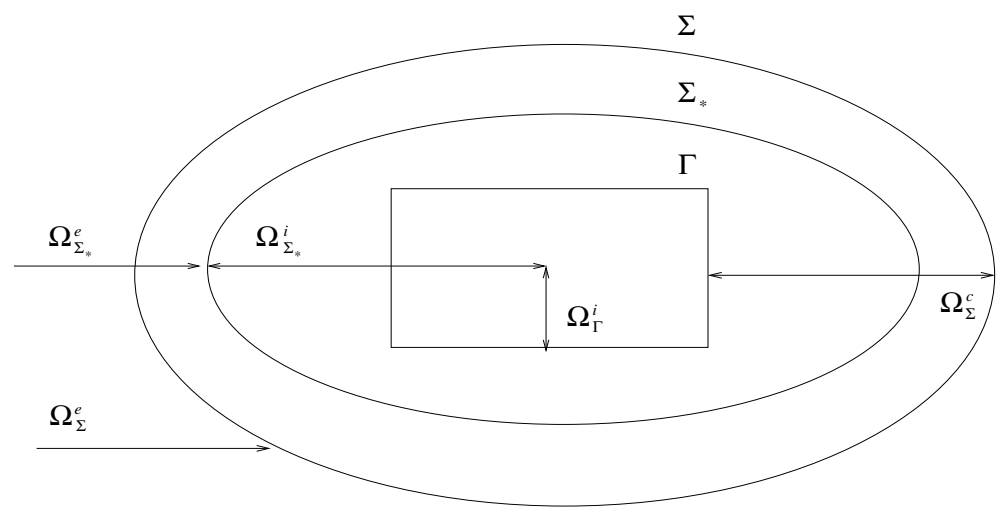

Figure 2. The geometry features.

\subsection{The Schwarz algorithm for the exterior Neumann problem}

More geometrical constructions and further notations are helpful for the clarity in the description of the Schwarz algorithm. Let $\Sigma_{*}$ be any closed smooth surface given in $\Omega_{\Sigma}^{c}$, then we denote by $\Omega_{\Sigma_{*}}^{c}$ the annular domain bordered by $\Gamma$ and $\Sigma_{*}$. Noticeably, $\Sigma_{*}$ induces a partition of the space into an interior domain $\Omega_{\Sigma_{*}}^{i}$ and an exterior domain $\Omega_{\Sigma_{*}}^{e}$. Unless explicitly contradicted, we assume in the subsequent that $\Gamma \cap \Sigma_{*}=\emptyset$ and $\Sigma_{*} \cap \Sigma=\emptyset$. We have that $\bar{\Omega}_{\Gamma}^{e}$ is the union of both $\bar{\Omega}_{\Sigma}^{c}$ and $\bar{\Omega}_{\Sigma_{*}}^{e}$ whose intersection is the annular domain located between $\Sigma$ and $\Sigma_{*}$ (see Fig. 2).

The purpose of the Schwarz method is the construction of a sequence $\left(w^{m}\right)_{m}$ approximating $u$. This is achieved following a recurrence. The terms $\left(w^{k}\right)_{0 \leq k \leq 2 m}$ being known, $w^{2 m+1}$ is computed by solving

$$
\begin{aligned}
-\Delta w^{2 m+1} & =0, & & \text { in } \Omega_{\Sigma_{*}}^{e}, \\
w^{2 m+1} & =w^{2 m}, & & \text { on } \Sigma_{*}, \\
\nabla w^{2 m+1} & =o\left(\frac{1}{|\boldsymbol{x}|}\right), & & \text { at infinity, }
\end{aligned}
$$

and $w^{2 m+2}$ is the solution of

$$
\begin{aligned}
-\Delta w^{2 m+2} & =0, & & \text { in } \Omega_{\Sigma}^{c}, \\
\partial_{\boldsymbol{n}} w^{2 m+2} & =g, & & \text { on } \Gamma, \\
w^{2 m+2} & =w^{2 m+1}, & & \text { on } \Sigma .
\end{aligned}
$$

Both boundary value problems have variational interpretation in the space $W^{1}\left(\Omega_{\Sigma_{*}}^{e}\right)$ for the first and in the space $H^{1}\left(\Omega_{\Sigma}^{c}\right)$ for the second. The theory developed by P.-L. Lions in [30] applies as well and yields the estimate

$$
\left\|u-w^{2 m+1}\right\|_{W^{1}\left(\Omega_{\Sigma_{*}}^{e}\right)}+\left\|u-w^{2 m}\right\|_{H^{1}\left(\Omega_{\Sigma}^{c}\right)} \leq C\left(w^{0}\right) \rho^{m}, \quad \forall m \in \mathbb{N} .
$$

The reduction factor $\rho \in\left[0,1\right.$ [ depends on the size of the overlapping regions $\left(\Omega_{\Sigma_{*}}^{e} \cap \Omega_{\Sigma}^{c}\right)$; it is lower for thicker overlapping region and faster is the convergence of the algorithm (see [43] for a relevant discussion). The efficiency of the Schwarz algorithm can be significantly increased by some judicious adaptations to the specific features of the exterior problems (taking profit of the integral representations). The boundary condition (12) on $\Sigma$ transmitted from $w^{2 m}$ to $w^{2 m+1}$ can be advantageously affected by exploiting the information contained 
in $\left(\partial_{\boldsymbol{n}} w^{2 m}\right)$ on $\Sigma_{*}$. It follows that, for the modified Schwarz algorithm, the problem (11)-(13) is changed into

$$
\begin{aligned}
-\Delta w^{2 m+1} & =0, & & \text { in } \Omega_{\Sigma_{*}}^{i} \text { and } \Omega_{\Sigma_{*}}^{e}, \\
{\left[\partial_{\boldsymbol{n}} w^{2 m+1}\right] } & =\partial_{\boldsymbol{n}} w^{2 m}, & & \text { on } \Sigma_{*}, \\
{\left[w^{2 m+1}\right] } & =w^{2 m}, & & \text { on } \Sigma_{*}, \\
\nabla w^{2 m+1} & =o\left(\frac{1}{|\boldsymbol{x}|}\right), & & \text { at infinity, }
\end{aligned}
$$

while the form of the value problem on $w^{2 m+2}$ remains unchanged, apart from the fact that the Dirichlet condition (16) is fed by the new $w^{2 m+1}$. Two points can be invoked to illustrate the power of the new approach. One is related to the time computational gain and the other to its convergence speed. The complexity for the calculation of $w^{2 m+1}$ is substantially reduced, since it is explicitly obtained by

$$
w^{2 m+1}(\boldsymbol{x})=\left(K_{\Sigma_{*}}\left(w_{\mid \Sigma_{*}}^{2 m}\right)-V_{\Sigma_{*}}\left(\partial_{\boldsymbol{n}} w_{\mid \Sigma_{*}}^{2 m}\right)\right)(\boldsymbol{x}), \quad \forall \boldsymbol{x} \in \mathbb{R}^{3} \backslash \Sigma_{*} .
$$

Compared to the standard Schwarz algorithm, an inversion of the exterior problem (11)-(13) is avoided at each step. Even more, $w^{2 m+1}$ is eliminated in the practice; indeed plugging (21) into the Dirichlet condition (16) on $w^{2 m+2}$, we obtain a sequence $\left(u^{m}\right)_{m}=\left(w^{2 m}\right)_{m}$ defined by the following induction: $u^{m}$ being known, we solve a Laplace equation in $\Omega_{\Sigma}^{c}$ with Neumann/Dirichlet conditions on $\Gamma / \Sigma$ that reads as: find $u^{m+1} \in H^{1}\left(\Omega_{\Sigma}^{c}\right)$ such that

$$
\begin{aligned}
-\Delta u^{m+1} & =0, & & \text { in } \Omega_{\Sigma}^{c} \\
\partial_{\boldsymbol{n}} u^{m+1} & =g, & & \text { on } \Gamma, \\
u^{m+1} & =K_{\Sigma_{*}}\left(u_{\mid \Sigma_{*}}^{m}\right)-V_{\Sigma_{*}}\left(\partial_{\boldsymbol{n}} u_{\mid \Sigma_{*}}^{m}\right), & & \text { on } \Sigma .
\end{aligned}
$$

Remark 2.3. The implementation of the modified Schwarz algorithm in MELINA is based on the formulation (22-24) (see [24]). As the interface $\Sigma$ is a closed surface, no restriction needs to be imposed on the initial function $u^{0}$ (or on $w^{0}$ ); so it can be chosen arbitrarily. Given that in our computation, the limit choice of the intermediary $\Sigma_{*}$ is provided by $\Gamma$, we prefer to start from $u^{0}=V_{\Sigma_{*}}(g)$ and we recommend it.

Remark 2.4 (Connection with the additive Schwarz method). The additive version of the Schwarz method, introduced in [12] for the bounded domains, is obtained by changing the fictitious condition (16) into $w_{\mid \Sigma}^{2 m+2}=w_{\mid \Sigma}^{2 m-1}$. This choice implies an uncoupling of both problems on $w^{2 m+1}$ and $w^{2 m+2}$. Notice that the alternating and additive algorithms yield to the same construction of the sequence $\left(u^{m}\right)_{m}$. This may be seen again by the integral representation of $w^{2 m+1}$. In fact, $w^{2 m+2}$ still satisfies equation (22), the Neumann condition (23) and the following Dirichlet condition:

$$
w^{2 m+2}=K_{\Sigma_{*}}\left(w_{\mid \Sigma_{*}}^{2 m-2}\right)-V_{\Sigma_{*}}\left(\partial_{\boldsymbol{n}} w_{\mid \Sigma_{*}}^{2 m-2}\right), \quad \text { on } \Sigma,
$$

from which we deduce that $u^{m}=w^{4 m}$. This attests that the alternating Schwarz method converges twice faster than the additive method for the unbounded domains.

Remark 2.5 (Connection with the Dirichlet-Neumann method). The limit case $\Sigma_{*}=\Sigma$ does not alter things, the modified Schwarz algorithm works as well. However, the Dirichlet condition prescribed to $w^{2 m+2}$ on $\Sigma$ needs to be precised a little more to avoid ambiguities (because of the discontinuity of $w^{2 m+1}$ on $\Sigma$ ); it should be understood as $w_{\mid \Sigma}^{2 m+2}=\left(w^{2 m+1}\right)_{\mid \Sigma}^{e}$. The resulting iterative procedure can be considered as a variant of the Dirichlet-Neumann technique, for which, in addition to the Neumann data $\left(\partial_{\boldsymbol{n}} w_{\mid \Sigma}^{2 m}\right)$, the information involved on $w_{\mid \Sigma}^{2 m}$ is transmitted to $w_{\mid \Sigma}^{2 m+1}$ on the boundary $\Sigma$. The sequence $\left(u^{m}\right)_{m}$ is constructed by the recurrence (22)-(23) 
while the boundary condition (24) becomes

$$
u^{m+1}=\left(\frac{1}{2} I_{\Sigma}+K_{\Sigma}\right)\left(u_{\mid \Sigma}^{m}\right)-V_{\Sigma}\left(\partial_{\boldsymbol{n}} u_{\mid \Sigma}^{m}\right), \quad \text { on } \Sigma .
$$

Remark 2.6. Remarks 2.4 and 2.5 show that our adaptation of the Schwarz process to the exterior problems results in a unified approach of several well known domain decomposition methods including both the multiplicative, the additive versions of the Schwarz method (see $[4,11,12,35]$ ) and the Dirichlet-Neumann iterative method (see [38]).

As far as the convergence analysis is concerned, the case $\Sigma_{*}=\Gamma$ plays an important role, since the convergence proof for an arbitrary $\Sigma_{*}$ is straightly obtained from this particular choice. It also seems a little more attractive, as the term depending on the simple layer potential has not to be updated during the iterations, inducing a reduction of the computational costs.

The variational formulation for $\Sigma_{*}=\Gamma$, is derived from (8) and relies on the decomposition $u^{m+1}=\mathcal{R}_{\Sigma}\left(K_{\Gamma}\left(u_{\mid \Gamma}^{m}\right)-V_{\Gamma}\left(u_{\mid \Gamma}^{m}\right)\right)+\underline{u}^{m+1}$, where $\underline{u}^{m+1} \in H_{0, \Sigma}^{1}\left(\Omega_{\Sigma}^{c}\right)$ is solution of

$$
\begin{aligned}
\int_{\Omega_{\Sigma}^{c}} \nabla \underline{u}^{m+1} \nabla v \mathrm{~d} \boldsymbol{x}= & \int_{\Gamma} g v \mathrm{~d} \gamma+\int_{\Omega_{\Sigma}^{c}} \nabla \mathcal{R}_{\Sigma}\left(K_{\Gamma}\left(u_{\mid \Gamma}^{m}\right)\right) \nabla v \mathrm{~d} \boldsymbol{x} \\
& -\int_{\Omega_{\Sigma}^{c}} \nabla \mathcal{R}_{\Sigma}\left(V_{\Gamma}\left(u_{\mid \Gamma}^{m}\right)\right) \nabla v \mathrm{~d} \boldsymbol{x}, \quad \forall v \in H_{0, \Sigma}^{1}\left(\Omega_{c}\right) .
\end{aligned}
$$

Achieving a finite element discretization, the sparsity and the symmetry of the stiffness matrix are fully restored, because of the uncoupling of $u_{\mid \Gamma}^{m+1}$ and $u_{\mid \Sigma}^{m+1}$, meanwhile the ellipticity is preserved. The algebraic system to be handled repeatedly, due to the recurrent updating of the Dirichlet data, can be solved efficiently by using a direct method like the Choleski technique, where the factorization of the matrix is done once for all in the pre-processing stage.

Before closing this subsection, we resume the case of an arbitrary $\Sigma_{*}$ to discuss the possible limit of the modified Schwarz method to figure out whether or not it provides the exact solution $u$ of (4). This inspired us the way to proceed for the proofs, since it gave us the idea of Lemma 2.7. Assume that $\left(w^{2 m}, w^{2 m+1}\right)$ converges toward $\left(w_{*}, w^{*}\right)$. Passing formally to the limit in both problems (17)-(20) and (22)-(24), we obtain that: $w^{*}$ is such that

$$
\begin{aligned}
-\Delta w^{*} & =0, & & \text { in } \Omega_{\Sigma_{*}}^{i} \text { and } \Omega_{\Sigma_{*}}^{e}, \\
{\left[\partial_{\boldsymbol{n}} w^{*}\right] } & =\partial_{\boldsymbol{n}} w_{*}, & & \text { on } \Sigma_{*}, \\
{\left[w^{*}\right] } & =w_{*}, & & \text { on } \Sigma_{*}, \\
\nabla w^{*} & =o\left(\frac{1}{|\boldsymbol{x}|}\right), & & \text { at infinity, }
\end{aligned}
$$

and: $w_{*}$ is solution of

$$
\begin{aligned}
-\Delta w_{*} & =0, & & \text { in } \Omega_{\Sigma}^{c}, \\
\partial_{\boldsymbol{n}} w_{*} & =g, & & \text { on } \Gamma, \\
w_{*} & =w^{*}, & & \text { on } \Sigma .
\end{aligned}
$$

Two configurations are possible, each one having its own analysis. 
We consider first that $\Sigma_{*} \cap \Sigma=\emptyset$ (the Jami-Lenoir method [22]).

Define $w_{* *}\left(\right.$ in $\left.\Omega_{\Sigma}^{i}\right)$ such that $w_{* *}=0$ in $\Omega_{\Sigma_{*}}^{i}$ and $w_{* *}=w_{*}$ in $\Omega_{\Sigma}^{i} \backslash \Omega_{\Sigma_{*}}^{i}$. Then it is easy to check that $\left(w^{*}-w_{* *}\right)$ is solution of the homogeneous Dirichlet-Poisson problem on $\Omega_{\Sigma}^{i}$. The trivial function is the unique solution, thus $w^{*}=w_{* *}$. In particular, we have that $w^{*}=w_{*}$ in $\Omega_{\Sigma}^{i} \backslash \Omega_{\Sigma_{*}}^{i}$. Setting

$$
w(\boldsymbol{x})= \begin{cases}w_{*}(\boldsymbol{x}), & \forall \boldsymbol{x} \in \Omega_{\Sigma}^{c} \\ w^{*}(\boldsymbol{x}), & \forall \boldsymbol{x} \in \Omega_{\Sigma_{*}}^{e}\end{cases}
$$

results in a coherent function in $\Omega_{\Gamma}^{e}$, and it is solution of the problem (1)-(3). Uniqueness produces that $w=u$.

The case where $\Sigma_{*}=\Sigma$ (the Johnson-Nédélec method [25]) proceeds as follows.

The Dirichlet condition prescribed to $w_{*}$ on $\Sigma$ says that $w_{* \mid \Sigma}=\left(w^{*}\right)_{\mid \Sigma}^{e}$ (see Rem. 2.5). Furthermore, an argumentation like that of $(25)$ yields that

$$
\frac{\left(w_{*}\right)_{\mid \Sigma}}{2}=K_{\Sigma}\left(w_{* \mid \Sigma}\right)-V_{\Sigma}\left(\partial_{\boldsymbol{n}} w_{* \mid \Sigma}\right)
$$

This results in $w^{*}=0$ in $\Omega_{\Sigma}^{i}$ and therefore, $\left(\partial_{\boldsymbol{n}} w^{*}\right)_{\mid \Sigma}=\left(\partial_{\boldsymbol{n}} w_{*}\right)_{\mid \Sigma}$. We deduce that $w$ defined by

$$
w(\boldsymbol{x})= \begin{cases}w_{*}(\boldsymbol{x}), & \forall \boldsymbol{x} \in \Omega_{\Sigma}^{c}, \\ w^{*}(\boldsymbol{x}), & \forall \boldsymbol{x} \in \Omega_{\Sigma}^{e},\end{cases}
$$

is solution of the boundary value problem (1)-(3) and consequently $w=u$.

The final conclusion is that for an arbitrary $\Sigma_{*}$ taken in $\Omega_{\Sigma}^{c}$, the limits of the alternating sequences allow the recovering of the exact solution of the exterior Laplace-Neumann problem when the convergence of the Schwarz algorithm is guaranteed.

\subsection{An analytical example}

To assess their liability to provide the exact solution, we present an analytical example using the classical Schwarz, the Dirichlet-Neumann and the modified Schwarz methods.

We use the spherical coordinates $(r, \theta, \varphi)$ in $\mathbb{R}^{3}$. We Consider that $\Gamma, \Sigma_{*}$ and $\Sigma$ are three spheres in $\mathbb{R}^{3}$ radii $1, R_{*}$ and $R$ respectively $\left(1 \leq R_{*} \leq R, R>1\right)$. We investigate the Laplace-Neumann problem set in $\Omega_{\Gamma}^{e}=\left\{\boldsymbol{x} \in \mathbb{R}^{3},|\boldsymbol{x}|>1\right\}$. The boundary data $g$ is chosen so that $u(r, \theta, \varphi)=r^{-k-1} Y_{k}^{n}(\theta, \varphi)$ is the exact solution, and $Y_{k}^{n},(k \in \mathbb{N},|n| \leq k)$ is the spherical harmonic of $k$-th order (see [44] for more details). The case $(k=0)$ is special, since both Dirichlet-Neumann and the modified Schwarz algorithms succeed to capture the exact solution $u(r, \theta, \varphi)=\frac{1}{r}$ in one iteration. Then it is excluded from the study and we assume that $k \geq 1$. An explicit construction of the sequence $\left(w^{m}\right)_{m}$ is possible by (22)-(24). From some symmetry considerations, $w^{m}$ has the following form $w^{m}(r, \theta, \varphi)=h^{m}(r) Y_{k}^{n}(\theta, \varphi)$ with

$$
h^{2 m}(r)=c_{2 m} r^{k}+d_{2 m} r^{-k-1}, \quad h^{2 m+1}(r)=b_{2 m+1} r^{-k-1} .
$$

We start by the classical Schwarz algorithm.

To be brief, we restrict the presentation to the analysis of the convergence of $w_{\mid \Omega_{\Sigma_{*}}^{e}}^{2 m+1}$. The convergence of the remaining quantities follows the same trends. Using the boundary conditions and achieving the overall computations provides

$$
\left(b_{2 m+3}-1\right)=\frac{1+\left(1+k^{-1}\right) R_{*}^{2 k+1}}{1+\left(1+k^{-1}\right) R^{2 k+1}}\left(b_{2 m+1}-1\right)=\rho(k)\left(b_{2 m+1}-1\right) .
$$

Under the mild assumption $\left(R_{*}<R\right)$, the classical Schwarz algorithm converges geometrically fast toward the exact solution $u$. The reducing factor $\rho(k)$ on the error depends on the size of the overlapping region 
$\left\{\boldsymbol{x}, R_{*} \leq|\boldsymbol{x}| \leq R\right\}$. The convergence of the Schwarz algorithm is slower for thin annular region. The critical point is reached when $\left(R_{*}=R\right)$, for which the convergence is not guaranteed anymore.

For the non-overlapping case $\left(R_{*}=R\right)$, the Dirichlet-Neumann procedure is recommended.

The corresponding boundary conditions to be exchanged across $\Sigma$ produce

$$
\left(b_{2 m+3}-1\right)=\frac{1-R^{2 k+1}}{1+\left(1+k^{-1}\right) R^{2 k+1}}\left(b_{2 m+1}-1\right)=\varrho(k)\left(b_{2 m+1}-1\right) .
$$

Although the convergence toward the exact solution $u$ is obtained, this approach suffers from some weakness. The reduction factor $\varrho(k)$ comes close to 1 for high values of $k$ (inducing a slowing of the convergence speed). Yet, these facts are known for bounded domains and that they still hold for the unbounded domains is not surprising.

Let us, now, skip to the modified Schwarz method for which $h^{2 m}$ and $h^{2 m+1}$ have rather the following form:

$$
h^{2 m}(r)=c_{2 m} r^{k}+d_{2 m} r^{-k-1}, \quad h^{2 m+1}(r)= \begin{cases}a_{2 m+1} r^{k}, & r \leq R_{*} \\ b_{2 m+1} r^{-k-1}, & r \geq R_{*}\end{cases}
$$

The computations, based on the transmission and the boundary conditions, show that $\left(b_{2 m+1}\right)_{m}$ obeys to the recurrence

$$
\left(b_{2 m+3}-1\right)=\frac{1}{1+\left(1+k^{-1}\right) R^{2 k+1}}\left(b_{2 m+1}-1\right)=\tau(k)\left(b_{2 m+1}-1\right) .
$$

The new version of the Schwarz algorithm is relevant, $\left(w^{2 m+1}\right)_{m}$ converges toward $u$ in $W^{1}\left(\Omega_{\Sigma_{*}}^{e}\right)$ and toward zero in $H^{1}\left(\Omega_{\Sigma_{*}}^{i}\right)$. Moreover, $\left(w^{2 m}\right)_{m}$ approaches $u$ in $H^{1}\left(\Omega_{\Sigma}^{e}\right)$ with the same rate $\tau(k)$. In addition to the economy brought about by the modification of the classical algorithm, another important effect of it is that the reduction factor does not depend on the location of the intermediary boundary $\Sigma_{*}$ (this is rigorously proved later in Lem. 2.7). The convergence rate is expected to remain constant, for $R_{*} \in[1, R]$. Notice also the strengthening of the convergence speed, compared to the classical alternating Schwarz method, since $\tau(k)<\rho(k)$ except for $R_{*}=1$, (i.e. $\Sigma_{*}=\Gamma$ ). Besides, the iterating algorithm still converges when $\Sigma_{*}=\Sigma$, and performs in general better than the Dirichlet-Neumann algorithm apart from a very special situation that is $R<\sqrt[3]{2}$ and for the lowest frequencies $k$.

\subsection{Geometrical convergence of the Schwarz algorithm}

We set the functional framework involved in the variational interpretation of the Schwarz algorithm. Let $V$ be the Hilbert space $H^{1}\left(\Omega_{\Gamma}^{i}\right) \times W^{1}\left(\Omega_{\Gamma}^{e}\right)$ endowed with the norm

$$
\|v\|_{V}=\left[\left(\frac{1}{|\Gamma|} \int_{\Gamma} v^{i} \mathrm{~d} \gamma\right)^{2}+\left\|\nabla v^{i}\right\|_{\mathrm{L}^{2}\left(\Omega_{\Gamma}^{i}\right)}^{2}+\left\|\nabla v^{e}\right\|_{\mathrm{L}^{2}\left(\Omega_{\Gamma}^{e}\right)}^{2}\right]^{\frac{1}{2}}
$$

where $v^{i}=v_{\mid \Omega_{\Gamma}^{i}}$ and $v^{e}=v_{\mid \Omega_{\Gamma}^{e}}$. The associated semi-norm is

$$
|v|_{V}=\left[\left\|\nabla v^{i}\right\|_{\mathrm{L}^{2}\left(\Omega_{\Gamma}^{i}\right)}^{2}+\left\|\nabla v^{e}\right\|_{\mathrm{L}^{2}\left(\Omega_{\Gamma}^{e}\right)}^{2}\right]^{\frac{1}{2}} .
$$

The recurrence algorithm makes a mathematical sense and results in two coherent sequences $\left(w^{2 m+1}\right)_{m} \subset V$ and $\left(w^{2 m}\right)_{m} \subset H^{1}\left(\Omega_{\Sigma}^{c}\right)$. The analysis begins by stating that the sequence $\left(u^{m}=w^{2 m}\right)_{m}$ does not depend on the intermediary boundary $\Sigma_{*}$. As a consequence, proving the convergence of the Schwarz algorithm for $\Sigma_{*}=\Gamma$, guarantees the convergence for any $\Sigma_{*}$, with the same speed.

Lemma 2.7. The sequence $\left(w^{2 m}\right)_{m}$ defined by the Schwarz algorithm (11)-(13) and (17)-(20) or, in other words, the sequence $\left(u^{m}\right)_{m}$ defined by the recurrence (22)-(24), is independent of $\Sigma_{*}$. 
Proof. We show that the Dirichlet condition (16) of $w^{2 m+2}$ on $\Sigma$ remains unchanged for arbitrary $\Sigma_{*}$. Let $\tilde{w}^{2 m+1}$ be defined such that

$$
\tilde{w}^{2 m+1}(\boldsymbol{x})= \begin{cases}w^{2 m+1}(\boldsymbol{x}), & \forall \boldsymbol{x} \in \Omega_{\Gamma}^{i} \cup \Omega_{\Sigma_{*} *}^{e}, \\ \left(w^{2 m+1}+w^{2 m}\right)(\boldsymbol{x}), & \forall \boldsymbol{x} \in \Omega_{\Sigma_{*}}^{i} \backslash \Omega_{\Gamma}^{i}\end{cases}
$$

We check that $\left(\left[\tilde{w}^{2 m+1}\right],\left[\partial_{\boldsymbol{n}} \tilde{w}^{2 m+1}\right]\right)=(0,0)$ on $\Sigma_{*}$, and we have that $\left(\left[\tilde{w}^{2 m+1}\right],\left[\partial_{\boldsymbol{n}} \tilde{w}^{2 m+1}\right]\right)=\left(w^{2 m}, g\right)$ on $\Gamma$. Therefore $\tilde{w}^{2 m+1}$ is the unique solution of the transmission problem across $\Gamma$. The integral representation results in

$$
\tilde{w}^{2 m+1}(\boldsymbol{x})=w^{2 m+1}(\boldsymbol{x})=\left(K_{\Gamma}\left(w_{\mid \Gamma}^{2 m}\right)-V_{\Gamma}(g)\right)(\boldsymbol{x}), \quad \forall \boldsymbol{x} \in \Sigma .
$$

Plugging this in (16) yields the desired result.

Likely, it is worth working in the space $V$ for the theoretical issues. Therefore, we extend $u \in W^{1}\left(\Omega_{\Gamma}^{e}\right)$ by zero in $\Omega_{\Gamma}^{i}$ to obtain a function in $V$. Similarly, $w^{2 m}$ is extended by zero in $\Omega_{\Gamma}^{i}$ and by $w^{2 m-1}$ in $\Omega_{\Gamma}^{e} \backslash \Omega_{\Sigma}^{c}$. The resulting function has no jump across $\Sigma$ and belongs to $V$ as soon as $w^{2 m-1} \in W^{1}\left(\Omega_{\Gamma}^{e}\right)$. For the sake of commodity, both extensions are still denoted $u$ and $w^{2 m}$ respectively. Using equations (1)-(3) and (17)-(20), we have that

$$
\begin{aligned}
-\Delta\left(w^{2 m+1}-u\right) & =0, & & \text { in } \Omega_{\Gamma}^{i} \text { and } \Omega_{\Gamma}^{e}, \\
{\left[\partial_{\boldsymbol{n}}\left(w^{2 m+1}-u\right)\right] } & =0, & & \text { on } \Gamma, \\
{\left[\left(w^{2 m+1}-u\right)\right] } & =w^{2 m}-u, & & \text { on } \Gamma, \\
\nabla\left(w^{2 m+1}-u\right) & =o\left(\frac{1}{|\boldsymbol{x}|}\right), & & \text { at infinity. }
\end{aligned}
$$

We deduce the variational relation (we set $V_{1}=W^{1}\left(\mathbb{R}^{3}\right)$ )

$$
\int_{\mathbb{R}^{3}} \nabla\left(w^{2 m+1}-w^{2 m}\right) \nabla v \mathrm{~d} \boldsymbol{x}=\int_{\Omega_{\Gamma}^{i} \cup \Omega_{\Gamma}^{e}} \nabla\left(u-w^{2 m}\right) \nabla v \mathrm{~d} \boldsymbol{x}, \quad \forall v \in V_{1} .
$$

Since $\left(w^{2 m+1}-w^{2 m}\right)$ belongs to $V_{1}$, we obtain that

$$
\left(w^{2 m+1}-w^{2 m}\right)=P_{V_{1}}\left(u-w^{2 m}\right)
$$

where $P_{V_{1}}$ is the orthogonal projection on $V_{1}$ with respect to the semi-norm $\left.\left.\right|_{V}\right|_{V}$ (recall that actually, it is a norm on $V_{1}$ equivalent to $\left.\|\cdot\|_{V}\right)$. The complementary identity, that is $\left(w^{2 m+2}-w^{2 m+1}\right)$ is the projection of $\left(u-w^{2 m}\right)$, relies on the space

$$
V_{2}=\left\{v=\left(v^{i}, v^{e}\right) \in V, \quad\left(v^{e}\right)_{\mid \Omega_{\Sigma}^{e}}=0\right\} .
$$

On one hand side $\left(w^{2 m+2}-w^{2 m+1}\right)$ belongs to $V_{2}$, and on the other hand side the following value problem is derived from (17)-(20) and (14)-(16)

$$
\begin{aligned}
-\Delta\left(w^{2 m+2}-w^{2 m+1}\right) & =-\Delta\left(u-w^{2 m+1}\right), & & \text { in } \Omega_{\Sigma}^{c}, \\
\partial_{\boldsymbol{n}}\left(w^{2 m+2}-w^{2 m+1}\right) & =\partial_{\boldsymbol{n}}\left(u-w^{2 m+1}\right), & & \text { on } \Gamma, \\
\left(w^{2 m+2}-w^{2 m+1}\right) & =0, & & \text { on } \Sigma .
\end{aligned}
$$

It can be reformulated variationally by

$$
\int_{\Omega_{\Gamma}^{i} \cup \Omega_{\Gamma}^{e}} \nabla\left(w^{2 m+2}-w^{2 m+1}\right) \nabla v \mathrm{~d} \boldsymbol{x}=\int_{\Omega_{\Gamma}^{i} \cup \Omega_{\Gamma}^{e}} \nabla\left(u-w^{2 m+1}\right) \nabla v \mathrm{~d} \boldsymbol{x}, \quad \forall v \in V_{2} .
$$


Taking into account that $\left(w^{2 m+2}\right)^{i}=u^{i}=0$ in $\Gamma$, implies that

$$
\left(w^{2 m+2}-w^{2 m+1}\right)=P_{V_{2}}\left(u-w^{2 m+1}\right)
$$

$P_{V_{2}}$ being the orthogonal projection on $V_{2}$ with respect to the norm $\|\cdot\|_{V}$. Putting together (29) and (30) yields the inductions

$$
\begin{aligned}
& \left(u-w^{2 m+2}\right)=\left(I-P_{V_{2}}\right)\left(I-P_{V_{1}}\right)\left(u-w^{2 m}\right), \\
& \left(u-w^{2 m+1}\right)=\left(I-P_{V_{1}}\right)\left(I-P_{V_{2}}\right)\left(u-w^{2 m-1}\right) .
\end{aligned}
$$

The proof that the error $\left\|u-w^{m}\right\|_{V}$ decreases toward zero can be obtained after checking that both operators $\left(I-P_{V_{1}}\right)$ and $\left(I-P_{V_{2}}\right)$ are contractions. To achieve this, we proceed like P.-L. Lions (see [30]). First we consider a function $\alpha \in \mathcal{D}\left(\mathbb{R}^{3}\right)(0 \leq \alpha \leq 1)$, supported in $\Omega_{\Gamma}^{i} \cup \bar{\Omega}_{\Sigma}^{c}$ with $\alpha=1$ in $\Omega_{\Gamma}^{i}$. Then, for any $v \in V$, we have that

$$
v=(1-\alpha) v+\alpha v=v_{1}+v_{2} \in V_{1}+V_{2}, \quad \int_{\Gamma} v^{i} \mathrm{~d} \gamma=\int_{\Gamma}\left(v_{2}\right)^{i} \mathrm{~d} \gamma
$$

As a result, there exists $\delta \geq 1$, depending only on $\alpha$, such that

$$
\left(\left|v_{1}\right|_{V}^{2}+\left|v_{2}\right|_{V}^{2}\right)^{\frac{1}{2}} \leq \delta|v|_{V}
$$

Lemma 2.8. For any $v \in V$, we have

$$
|v|_{V} \leq \delta\left(\left|P_{V_{1}} v\right|_{V}^{2}+\left|P_{V_{2}} v\right|_{V}^{2}\right)^{\frac{1}{2}}
$$

where $\delta$ is the stability constant given by (31).

Proof. Let $v \in V$, we have that

$$
|v|_{V}^{2}=\int_{\Omega_{\Gamma}^{i} \cup \Omega_{\Gamma}^{e}} \nabla v \nabla v_{1} \mathrm{~d} \boldsymbol{x}+\int_{\Omega_{\Gamma}^{i} \cup \Omega_{\Gamma}^{e}} \nabla v \nabla v_{2} \mathrm{~d} \boldsymbol{x} .
$$

The definition of $P_{V_{1}}$ and $P_{V_{2}}$ leads to

$$
|v|_{V}^{2}=\int_{\Omega_{\Gamma}^{i} \cup \Omega_{\Gamma}^{e}} \nabla\left(P_{V_{1}} v\right) \nabla v_{1} \mathrm{~d} \boldsymbol{x}+\int_{\Omega_{\Gamma}^{i} \cup \Omega_{\Gamma}^{e}} \nabla\left(P_{V_{2}} v\right) \nabla v_{2} \mathrm{~d} \boldsymbol{x} .
$$

Cauchy-Schwarz inequality ends to

$$
|v|_{V}^{2} \leq\left|P_{V_{1}} v\right|_{V}\left|v_{1}\right|_{V}+\left|P_{V_{2}} v\right|_{V}\left|v_{2}\right|_{V} \leq\left(\left|P_{V_{1}} v\right|_{V}^{2}+\left|P_{V_{2}} v\right|_{V}^{2}\right)^{\frac{1}{2}}\left(\left|v_{1}\right|_{V}^{2}+\left|v_{2}\right|_{V}^{2}\right)^{\frac{1}{2}}
$$

The stability (31) completes the proof.

Remark 2.9. Since $\int_{\Gamma}\left(v_{i}-\left(P_{V_{2}} v\right)_{i}\right) \mathrm{d} \gamma=0$, then

$$
\|v\|_{V} \leq \delta^{\prime}\left(\left|P_{V_{1}} v\right|_{V}^{2}+\left\|P_{V_{2}} v\right\|_{V}^{2}\right)^{\frac{1}{2}}
$$

with $\delta^{\prime}=\max (1, \delta)$.

Proposition 2.10. The operators $\left(I-P_{V_{1}}\right)\left(I-P_{V_{2}}\right)$ and $\left(I-P_{V_{2}}\right)\left(I-P_{V_{1}}\right)$ are contracting with respect to the semi norm $|\cdot|_{V}$, i.e. there exists a constant $\tau \in[0,1[$, such that $: \forall v \in V$,

$$
\begin{aligned}
& \left|\left(I-P_{V_{2}}\right)\left(I-P_{V_{1}}\right) v\right|_{V} \leq \tau|v|_{V} \\
& \left|\left(I-P_{V_{1}}\right)\left(I-P_{V_{2}}\right) v\right|_{V} \leq \tau|v|_{V} .
\end{aligned}
$$


Proof. We follow the same proof of Theorem I.1 of [30]. It is reproduced only for self-consistency. Applying (32) to $\left(I-P_{V_{1}}\right) v$, yields that

$$
\left|\left(I-P_{V_{1}}\right) v\right|_{V} \leq \delta\left|P_{V_{2}}\left(I-P_{V_{1}}\right) v\right|_{V}, \quad \forall v \in V .
$$

The triangular equality provides

$$
\left|\left(I-P_{V_{1}}\right) v\right|_{V}^{2}=\left|\left(I-P_{V_{2}}\right)\left(I-P_{V_{1}}\right) v\right|_{V}^{2}+\left|P_{V_{2}}\left(I-P_{V_{1}}\right) v\right|_{V}^{2}, \quad \forall v \in V .
$$

Then

$$
\left|\left(I-P_{V_{1}}\right) v\right|_{V}^{2} \geq\left|\left(I-P_{V_{2}}\right)\left(I-P_{V_{1}}\right) v\right|_{V}^{2}+\frac{1}{\delta^{2}}\left|\left(I-P_{V_{1}}\right) v\right|_{V}^{2}, \quad \forall v \in V,
$$

from which we deduce (34) with $\tau=\left(1-\frac{1}{\delta^{2}}\right)^{\frac{1}{2}}<1$. Estimate (35) is proved in the same way.

Remark 2.11. Using, in the previous proof, the estimate (33) instead of (32) shows that the operators of iterations are also contractions with respect to the full norm $\|\cdot\|_{V}$, that is

$$
\begin{aligned}
& \left\|\left(I-P_{V_{2}}\right)\left(I-P_{V_{1}}\right) v\right\|_{V} \leq \tau^{\prime}\|v\|_{V}, \\
& \left\|\left(I-P_{V_{1}}\right)\left(I-P_{V_{2}}\right) v\right\|_{V} \leq \tau^{\prime}\|v\|_{V},
\end{aligned}
$$

with $\tau^{\prime}=\left(1-\frac{1}{\delta^{\prime 2}}\right)^{\frac{1}{2}}<1$.

The consequence of Proposition 2.10 is that the approximating sequence $\left(u^{m}\right)_{m}$ converges toward the exact solution $u$ of the Laplace-Neumann.

Theorem 2.12. The Schwarz algorithm converges with a geometrical rate: there exists $\tau \in[0,1[$ such that

$$
\left|u-w^{m}\right|_{V} \leq C\left(u^{0}\right) \tau^{m}, \quad \forall m \in \mathbb{N} .
$$

Therefore, it holds that

$$
\left|u-u^{m}\right|_{H^{1}\left(\Omega_{\Sigma}^{c}\right)} \leq C\left(u^{0}\right) \tau^{m}, \quad \forall m \in \mathbb{N} .
$$

Remark 2.13. From Remark 2.11 we have that

$$
\left\|u-w^{m}\right\|_{V} \leq C\left(u^{0}\right)\left(\tau^{\prime}\right)^{m}, \quad \forall m \in \mathbb{N} .
$$

This tells that $\left\|w^{2 m+1}\right\|_{H^{1}\left(\Omega_{\Gamma}^{i}\right)}$ decays to zero like $C\left(u^{0}\right)\left(\tau^{\prime}\right)^{m}$ which is in agreement with the expectations.

Remark 2.14. Theorem 2.12 indicates that the convergence of the Schwarz algorithm is ensured for a fictitious boundary $\Sigma$ arbitrarily chosen provided that $\Sigma \cap \Gamma=\emptyset$. The convergence speed depends on the size of the overlapping region. It should be higher for thicker $\Omega_{\Sigma}^{c}$. This is already seen for the analytical tests and will be confirmed by some relevant numerical examples in Section 6 .

\section{The Laplace-Dirichlet Problem in $\mathbb{R}^{3}$}

The Laplace-Dirichlet problem reads in the same words as the Laplace-Neumann equation, excepted for the boundary condition prescribed on $\Gamma$. The data $g$ being given in $H^{\frac{1}{2}}(\Gamma)$, we have to: find $u$ satisfying

$$
\begin{aligned}
-\Delta u & =0, & & \text { in } \Omega_{\Gamma}^{e}, \\
u & =g, & & \text { on } \Gamma, \\
\nabla u & =o\left(\frac{1}{|\boldsymbol{x}|}\right), & & \text { at infinity. }
\end{aligned}
$$


Let us consider the subspace of $W^{1}\left(\Omega_{\Gamma}^{e}\right)$, after incorporating the homogeneous Dirichlet boundary condition

$$
W_{0}^{1}\left(\Omega_{\Gamma}^{e}\right)=\left\{v \in W^{1}\left(\Omega_{\Gamma}^{e}\right), \quad v_{\mid \Gamma}=0\right\},
$$

and let $\mathcal{R}_{\Gamma}$ be a stable extension from $H^{\frac{1}{2}}(\Gamma)$ into $W^{1}\left(\Omega_{\Gamma}^{e}\right)$ (see [10]). The variational formulation is based on the introduction of an auxiliary unknown by the means of the decomposition $u=\underline{u}+\mathcal{R}_{\Gamma} g$, so that $\underline{u} \in W_{0}^{1}\left(\Omega_{\Gamma}^{e}\right)$ is solution of

$$
\int_{\Omega_{\Gamma}^{e}} \nabla \underline{u} \nabla v \mathrm{~d} \boldsymbol{x}=-\int_{\Omega_{\Gamma}^{e}} \nabla\left(\mathcal{R}_{\Gamma} g\right) \nabla v \mathrm{~d} \boldsymbol{x}, \quad \forall v \in W_{0}^{1}\left(\Omega_{\Gamma}^{e}\right) .
$$

Writing down the Schwarz algorithm is realized as for the Neumann problem. The only difference is concerned with the fictitious boundary condition imposed on $\Sigma$; which is expressed by a Neumann condition. Let $w^{0} \in H^{1}\left(\Omega_{\Sigma}^{c}\right)$ be an initial guess, then $w^{2 m+1}$ solves the transmission problem (17)-(20) and $w^{2 m+2}$ is such that

$$
\begin{aligned}
-\Delta w^{2 m+2} & =0, & & \text { in } \Omega_{\Sigma}^{c}, \\
w^{2 m+2} & =g, & & \text { on } \Gamma, \\
\partial_{\boldsymbol{n}} w^{2 m+2} & =\partial_{\boldsymbol{n}} w^{2 m+1}, & & \text { on } \Sigma .
\end{aligned}
$$

After eliminating $w^{2 m+1}$, the Schwarz algorithm comes out with the construction of the sequence $\left(u^{m}\right)_{m}=\left(w^{2 m}\right)_{m}:$ find $u^{m+1} \in H^{1}\left(\Omega_{\Sigma}^{c}\right)$ such that

$$
\begin{aligned}
-\Delta u^{m+1} & =0, & & \text { in } \Omega_{\Sigma}^{c}, \\
u^{m+1} & =g, & & \text { on } \Gamma, \\
\partial_{\boldsymbol{n}} u^{m+1} & =\partial_{\boldsymbol{n}}\left[K_{\Sigma_{*}}\left(u_{\mid \Sigma_{*}}^{m}\right)\right]-\partial_{\boldsymbol{n}}\left[V_{\Sigma_{*}}\left(\partial_{\boldsymbol{n}} u_{\mid \Sigma_{*}}^{m}\right)\right], & & \text { on } \Sigma .
\end{aligned}
$$

The sequence $\left(u^{m}\right)_{m}$ being independent of the location of $\Sigma_{*}$ explains plainly why we restrict ourselves to the case $\Sigma_{*}=\Gamma$, for which the variational problem reads as: find $u^{m+1}=\underline{u}^{m+1}+\mathcal{R}_{\Gamma} g \in H^{1}\left(\Omega_{\Sigma}^{c}\right)$ satisfying

$$
\begin{aligned}
\int_{\Omega_{\Sigma}^{c}} \nabla \underline{u}^{m+1} \nabla v \mathrm{~d} \boldsymbol{x}= & -\int_{\Omega_{\Gamma}^{c}} \nabla\left(\mathcal{R}_{\Gamma} g\right) \nabla v \mathrm{~d} \boldsymbol{x} \\
& +\int_{\Omega_{\Gamma}^{c}} \nabla\left[K_{\Sigma_{*}}\left(u_{\mid \Sigma_{*}}^{m}\right)-V_{\Sigma_{*}}\left(\partial_{\boldsymbol{n}} u_{\mid \Sigma_{*}}^{m}\right)\right] \nabla v \mathrm{~d} \boldsymbol{x}, \quad \forall v \in H_{0}^{1}\left(\Omega_{\Sigma}^{c}\right) .
\end{aligned}
$$

Remark 3.1. Why not a Dirichlet condition on $\Sigma$ ?

There is a case which justifies why we do not impose a fictitious Dirichlet condition $w^{2 m+2}=w^{2 m+1}$ on $\Sigma$. The reason is based on an analytical example, for which the Dirichlet and the Neumann conditions are compared. We resume the geometrical description of Section 2.2. We choose $g=Y_{k}^{n}(\theta, \varphi)$ so that the exact solution is still $u(r, \theta, \varphi)=r^{-k-1} Y_{k}^{n}(\theta, \varphi)$. When a Dirichlet condition $w_{\mid \Sigma}^{2 m+2}=w_{\mid \Sigma}^{2 m+1}$ is taken, the radial functions $h^{2 m}$ and $h^{2 m+1}$ have the form of (27) with the following recurrence formula

$$
\left(b_{2 m+3}-1\right)=\frac{1}{1-R^{2 k+1}}\left(b_{2 m+1}-1\right)=\tau^{\prime}(k)\left(b_{2 m+1}-1\right) .
$$

The convergence of the sequence $\left(b_{2 m+1}\right)_{m}$ toward 1 is not guaranteed, unless $R>\sqrt[2 k+1]{2}$. Actually, for arbitrary Dirichlet data, the iterative process converges to the solution $u$ when the annular domain $\Omega_{\Sigma}^{c}$ has a minimal thickness $(R>\sqrt[3]{2})$.

Let us now turn to the case where $w^{2 m+2}$ is subjected to a Neumann condition $\partial_{\boldsymbol{n}} w_{\mid \Sigma}^{2 m+2}=\partial_{\boldsymbol{n}} w_{\mid \Sigma}^{2 m+1}$, the relations allowing the full computation of the sequence $\left(w^{m}\right)_{m}$ are exactly the same as (28). They conclude to the geometrical convergence of the Schwarz algorithm without any assumption on the size of $\Omega_{\Sigma}^{c}$. 
Again the convergence of the iterating Schwarz procedure (the convergence of $\left(u^{m}\right)_{m}$ toward $u_{\mid \Omega_{\Sigma}^{c}}$ with respect to the $H^{1}\left(\Omega_{\Sigma}^{c}\right)$ norm) for the Laplace-Dirichlet problem comes under the variational theory by P.-L. Lions (see [30]). Since they are close to the developments exposed for the Laplace-Neumann problem we do not reproduce them. The readers can find the details of the geometrical convergence study in the thesis of F. Jelassi (see [24]).

Theorem 3.2. The sequence $\left(u^{m}\right)_{m}$ defined by the Schwarz algorithm converges geometrically fast toward $u_{\mid \Omega_{\Sigma}^{c}}$ : there exists a real number $\tau \in[0,1[$ such that

$$
\left\|u-u^{m}\right\|_{H^{1}\left(\Omega_{\Sigma}^{c}\right)} \leq C\left(u^{0}\right) \tau^{m}, \quad \forall m \in \mathbb{N} .
$$

\section{The Laplace PRoBlem in $\mathbb{R}^{2}$}

We give some hints about the modifications to be added to the Laplace problem as well as to the integral representation formulas, in the two-dimensional case. First of all, the boundary condition at infinity expresses that only the boundedness is enforced (see $[13,23,44]$ )

$$
u=O(1) \quad \text { at infinity. }
$$

That recalled, the functional framework should be reset. The appropriate Sobolev space of work is determined by a two-dimensional specific weight

$$
W^{1}\left(\Omega_{\Gamma}^{e}\right)=\left\{v ; \frac{v}{\sqrt{1+|\boldsymbol{x}|^{2}} \log \left(2+|\boldsymbol{x}|^{2}\right)} \in L^{2}\left(\Omega_{\Gamma}^{e}\right), \quad \nabla v \in \mathrm{L}^{2}\left(\Omega_{\Gamma}^{e}\right)\right\} .
$$

The fundamental difference with the three-dimensional case is that all constant-functions belong to $W^{1}\left(\Omega_{\Gamma}^{e}\right)$; the immediate consequence is that the semi-norm $|\cdot|_{W^{1}\left(\Omega_{\Gamma}^{e}\right)}$ is not a norm any longer. The well posedness of the Neumann-Laplace problem (1)-(2) and (45) requires the assumption $\int_{\Gamma} g \mathrm{~d} \Gamma=0$ for the existence, and the spurious modes elimination for the uniqueness. We work, therefore, in the quotiented subspace

$$
W_{\Gamma}^{1}\left(\Omega_{\Gamma}^{e}\right)=\left\{v \in W^{1}\left(\Omega_{\Gamma}^{e}\right) ; \quad \int_{\Gamma} v \mathrm{~d} \Gamma=0\right\},
$$

endowed with the semi-norm $|\cdot|_{W^{1}\left(\Omega_{\Gamma}^{e}\right)}$ which becomes again a norm owing to the Hardy inequality (see $[27,28]$ ). The associated weak problem reads in the same terms as (4). The integral representation, fitting to the two dimensions, is given by

$$
u(\boldsymbol{x})=C+\int_{\Gamma} \varphi(\cdot) \partial_{\boldsymbol{n}} G(\boldsymbol{x}-\cdot) \mathrm{d} \gamma-\int_{\Gamma} g(\cdot) G(\boldsymbol{x}-\cdot) \mathrm{d} \gamma, \quad \forall \boldsymbol{x} \in \Omega_{\Gamma}^{e} .
$$

The Green function is given by $G(\boldsymbol{x})=-\frac{1}{2 \pi} \log |\boldsymbol{x}|$. Notice that the condition $\int_{\Gamma} g \mathrm{~d} \Gamma=0$, makes the constant $C$ in (46) to be the limit of the solution $u$ at infinity, meaning that $C=\lim _{|\boldsymbol{x}| \rightarrow \infty} u(\boldsymbol{x})$.

The description of the Schwarz method is restricted to the case where $\Sigma_{*}$ coincides with $\Gamma$. General configurations are handled by Lemma 2.7. The first subproblem on $w^{2 m+1}$, is composed of equations (17)-(19) and the condition (45) at infinity. The second one on $w^{2 m+2}$, is provided by equations (14)-(16). The variational interpretation is considered on the space $V=H^{1}\left(\Omega_{\Gamma}^{i}\right) \times W^{1}\left(\Omega_{\Gamma}^{e}\right)$ endowed with the norm

$$
\|v\|_{V}=\left[\left(\frac{1}{|\Gamma|} \int_{\Gamma} v^{i} \mathrm{~d} \gamma\right)^{2}+\left\|\nabla v^{i}\right\|_{\mathrm{L}^{2}\left(\Omega_{\Gamma}^{i}\right)}^{2}+\left(\frac{1}{|\Gamma|} \int_{\Gamma} v^{e} \mathrm{~d} \gamma\right)^{2}+\left\|\nabla v^{e}\right\|_{\mathrm{L}^{2}\left(\Omega_{\Gamma}^{e}\right)}^{2}\right]^{\frac{1}{2}} .
$$

At each step with odd iteration number, we solve the variational problem corresponding to (17)-(19) and (45) to obtain a unique solution $w^{2 m+1} \in H^{1}\left(\Omega_{\Gamma}^{i}\right) \times W_{\Gamma}^{1}\left(\Omega_{\Gamma}^{e}\right)$. For the step with even iteration number, the weak form 
of equations (14)-(16) is still well posed in $H^{1}\left(\Omega_{\Sigma}^{c}\right)$ and has only one solution $w^{2 m+2} \in H^{1}\left(\Omega_{\Sigma}^{c}\right)$. Also, we need to split the space $V$ into the sum $V_{1}+V_{2}$ for the convergence study issues. Compared to the three-dimensions, the space $V_{1}$ is changed to

$$
V_{1}=\left\{v \in W^{1}\left(\mathbb{R}^{2}\right) ; \quad \int_{\Gamma} v \mathrm{~d} \gamma=0\right\},
$$

while $V_{2}$ is kept as it is defined in three dimensions. We observe the basic facts that

$$
\|v\|_{V_{1}}=\left[\|\nabla v\|_{\mathrm{L}^{2}\left(\mathbb{R}^{2}\right)}+\left(\frac{1}{|\Gamma|} \int_{\Gamma} v^{e} \mathrm{~d} \gamma\right)^{2}\right]^{\frac{1}{2}}
$$

is a norm on $V_{1}$ which is equivalent to $\|\cdot\|_{V}$ and that

$$
\|v\|_{V_{2}}=\left[\left(\frac{1}{|\Gamma|} \int_{\Gamma} v^{i} \mathrm{~d} \gamma\right)^{2}+\left\|\nabla v^{i}\right\|_{L^{2}\left(\Omega_{\Gamma}^{i}\right)}^{2}+\left\|\nabla v^{e}\right\|_{L^{2}\left(\Omega_{\Gamma}^{e}\right)}^{2}\right]^{\frac{1}{2}}
$$

determines a norm on $V_{2}$, equivalent to $\|\cdot\|_{V}$. The corresponding inner products allows us to define the orthogonal projections $P_{V_{1}}$ and $P_{V_{2}}$ on $V_{1}$ and $V_{2}$. That (29) and (30) still hold is verified by checking that the same methodology still works. In consequence, all the results proved in the three dimensional case are valid. We refer to [24] for exhaustive details.

Switching to the practical aspects, we discuss how the algorithm is implemented. The recurrence formula on $\left(u^{m}=w^{2 m}\right)_{m}$ is exhibited by eliminating $w^{2 m+1}$ from the process by using the integral representation

$$
w^{2 m+1}(\boldsymbol{x})=c^{m}+K_{\Gamma}\left(w_{\mid \Gamma}^{2 m}\right)(\boldsymbol{x})-V_{\Gamma} g(\boldsymbol{x}), \quad \forall \boldsymbol{x} \notin \Gamma .
$$

The constant $c^{m}$ ensures that $\int_{\Gamma} w_{e}^{2 m+1} \mathrm{~d} \gamma=0$. This results in the following construction of $\left(u^{m}\right)_{m} ; u^{m}$ satisfies the Laplace equation (22), the Neumann condition (23) on $\Gamma$, and the artificial Dirichlet condition

$$
u^{m+1}=c^{m}+K_{\Gamma}\left(u_{\mid \Gamma}^{m}\right)(\boldsymbol{x})-V_{\Gamma} g(\boldsymbol{x}), \quad \text { on } \Sigma .
$$

Actually, in our programming we proceed in a slight different way. At each iteration, the constant $c^{m}$ is ignored, then an intermediary solution of the Neumann-Dirichlet-Laplace problem is computed, say $\tilde{u}^{m}$. At convergence, we obtain $u^{m}$ by subtracting a constant from $\tilde{u}^{m}$ in order to enforce $\int_{\Gamma} u^{m+1} \mathrm{~d} \gamma=0$.

\section{Some EXTEnsions}

The Schwarz algorithm can be successfully extended to handle many exterior problems, such as the viscous fluid flow around an obstacle governed by the Stokes and/or the Navier-Stokes equations (see [14,40]). Deriving a relevant outflow condition on the fictitious boundary of the truncated computational domain is an up-to-date question. Our algorithm may help to clarify such a point, at least for some special geometries. It may, also, be a performing computational tool of the displacement, stress and strain tensors for linear elastostatical problems in unbounded structures. We can go forth in the enumeration of interesting examples of physical systems, but we stop it here and we refer to the Ph.D. work of F. Jelassi for further developments, in particular for the eddy currents in electro-technical models (see [24]). Because of the lack of space, we only describe how to apply the Schwarz method for the Laplace problem with a source term and how to rewrite it in a half space.

\subsection{How to handle the source terms?}

In the theory detailed above, we did not take into account possible volume sources. Their presence in many physical models may cause some trouble in the statement of the Schwarz algorithm, and need to be clarified. 
The variable $u$ may describe an electrostatic potential created, in part, by volume charges. These sources lay, most often, in a located region and are represented by a function $f \in L^{2}\left(\Omega_{\Gamma}^{e}\right)$, compactly supported. The partial differential equation (1) is modified to

$$
-\Delta u=f, \quad \text { in } \Omega_{\Gamma}^{e},
$$

the Neumann condition and the infinity condition are not changed. The sequence $\left(u^{m}\right)_{m}$ provided by the Schwarz procedure, is calculated by recurrently solving the Poisson problem on the truncated domain: find $u^{m+1} \in H^{1}\left(\Omega_{\Sigma}^{c}\right)$ such that

$$
\begin{aligned}
-\Delta u^{m+1} & =f, \quad \text { in } \Omega_{\Sigma}^{c}, \\
\partial_{\boldsymbol{n}} u^{m+1} & =g, \quad \text { on } \Gamma, \\
u^{m+1} & =f_{\Sigma_{*}} \star G+K_{\Sigma_{*}}\left(u_{\mid \Sigma_{*}}^{m}\right)-V_{\Sigma_{*}}\left(\partial_{\boldsymbol{n}} u_{\mid \Sigma_{*}}^{m}\right), \quad \text { on } \Sigma .
\end{aligned}
$$

The symbol $\star$ stands for the convolution operator. A particular care should be paid to the determination of the function $f_{\Sigma_{*}}=\chi_{\Omega_{\Sigma_{*}}^{e}} f$, which is the trivial extension of $f_{\mid \Omega_{\Sigma_{*}}^{e}}$ to the whole space. Interpreting the algorithm as a domain decomposition iterative solver can be realized in the same spirit as when $f=0$. This allows us to establish the convergence of $\left(u^{m}\right)_{m}$ toward $u \in H^{1}\left(\Omega_{\Sigma}^{c}\right)$ geometrically fast.

\subsection{How to express the Schwarz method in the half space?}

We continue with the Poisson problem set in an unbounded domain which is assumed to be a part of the half space. The purpose is to explain how, by the means of the method of images (also called the reflexion method, see $[19,23]$ ), we can formulate the Schwarz scheme in a form liable to scientific implementations. Let then $\Omega^{e}$ and $\Omega^{i}$ be a partition of the upper half space $\mathbb{R}_{+}^{3}=\left\{\boldsymbol{x} \in \mathbb{R}^{3} ; x_{3}>0\right\}, \Omega^{i}$ is bounded and $\Gamma$ stands for the common boundary to both sub-domains. We denote by $\Gamma^{0}$ the portion of the boundary $\partial \Omega^{e}$ located in the plan $x_{3}=0$. The problem we are concerned with consists in: finding u such that

$$
\begin{aligned}
-\Delta u & =0, & & \text { in } \Omega^{e}, \\
\partial_{\boldsymbol{n}} u & =g, & & \text { on } \Gamma, \\
u & =0, & & \text { on } \Gamma^{0}, \\
\nabla u & =o\left(\frac{1}{|\boldsymbol{x}|}\right), & & \text { at infinity. }
\end{aligned}
$$

Notice that, the method of images is based on symmetrization techniques, odd or even according to the nature of the boundary condition on $\Gamma^{0}$, allows to derive some integral representation formulas adapted to the half space. The solution $u$ may be expressed as follows

$$
u(\boldsymbol{x})=\int_{\Gamma} u_{\mid \Gamma}(\cdot) \partial_{\boldsymbol{n}} G_{D}(\boldsymbol{x}, \cdot) \mathrm{d} \gamma-\int_{\Gamma} g(\cdot) G_{D}(\boldsymbol{x}, \cdot) \mathrm{d} \gamma, \quad \forall \boldsymbol{x} \in \Omega^{e} .
$$

The Green function $G_{D}$ is defined to be (see [23])

$$
G_{D}(\boldsymbol{x}, \boldsymbol{y})=\frac{1}{4 \pi|\boldsymbol{x}-\boldsymbol{y}|}-\frac{1}{4 \pi\left|\boldsymbol{x}-\boldsymbol{y}_{-}\right|}, \quad \forall \boldsymbol{x}, \boldsymbol{y} \in \mathbb{R}_{+}^{3}, \quad \boldsymbol{x} \neq \boldsymbol{y},
$$

where $\boldsymbol{y}_{-}=\left(y_{1}, y_{2},-y_{3}\right)$ is the point found by reflecting $\boldsymbol{y}=\left(y_{1}, y_{2}, y_{3}\right)$ across the $\left(x_{1}, x_{2}\right)$-plan, $\boldsymbol{y}_{-}$is said to be the image of $\boldsymbol{y}$. Writing down the Schwarz algorithm is based on a fictitious boundary $\Sigma$ surrounding the obstacle $\Gamma$. The Poisson equation, to be solved repeatedly, is set on the bounded domain $\Omega^{c}$ (the truncated domain delimited by $\Gamma, \Sigma$ and $\left.\Gamma^{0} \cap \Omega^{c}\right)$; it is composed of equations (47)-(49) and the artificial Dirichlet condition,

$$
u^{m+1}(\boldsymbol{x})=\int_{\Gamma} u_{\mid \Gamma}^{m}(\cdot) \partial_{\boldsymbol{n}} G_{D}(\boldsymbol{x}, \cdot) \mathrm{d} \gamma-\int_{\Gamma} g(\cdot) G_{D}(\boldsymbol{x}, \cdot) \mathrm{d} \gamma, \quad \forall \boldsymbol{x} \in \Sigma .
$$


The convergence of the algorithm, with a geometrical rate, can be stated following the lines exposed above (see [24], for details).

Remark 5.1. The Dirichlet condition on the boundary $\Gamma^{0}$ may be replaced by the Neumann condition $\left(\partial_{\boldsymbol{n}} u=0\right)$. Only some minor modifications have to be introduced. Among them, a suitable adaptation of the Green function should be considered

$$
G_{N}(\boldsymbol{x}, \boldsymbol{y})=\frac{1}{4 \pi|\boldsymbol{x}-\boldsymbol{y}|}+\frac{1}{4 \pi\left|\boldsymbol{x}-\boldsymbol{y}_{-}\right|}, \quad \forall \boldsymbol{x}, \boldsymbol{y} \in \mathbb{R}_{+}^{3}, \quad \boldsymbol{x} \neq \boldsymbol{y} .
$$

\section{A BRIEF NUMERICAL DISCUSSION}

The observations made, after the analytical calculations presented above, need to be validated by a numerical investigation. We consider some examples to check the reliability of our approach, (combined with a finite element procedure) and to illustrate the geometrical convergence rate of the Schwarz algorithm in the discrete context. A more systematic study of the Schwarz approach for additional models can be found in [24]. Let us mention that the overall finite element calculations use the Code MELINA (developed by the team of D. Martin at the University of Rennes I, see [34]) in which F. Jelassi added the specific procedures necessary to the Schwarz algorithm. All the numerical tests are realized in a simple precision calculations and we start up the Schwarz algorithm from the initial datum $u^{0}$ as indicated in Remark 2.3.

Before discussions, we indicate that the overall curves, plotted below, depict the variation of the relative gap between two consecutive iterates (computed by the Schwarz algorithm) with respect to the iterations number. Studying these curves so as to draw some informative conclusions about the reliability of the iterative method has become a classical methodology. To illustrate the accuracy of the Schwarz solution $u^{S c}=u^{m^{*}}$ (the one obtained after the convergence has been established, at iteration $\left.m^{*}\right)$, we give the measure of the error $\left(u-u^{S c}\right)$ for all the tests addressed here. When the exact solution $u$ is not available to us, we compare $u^{S c}$ to the coupled solution $u^{C o}$, obtained by solving the (FEM/BEM) problem (8), which is well known to be a satisfactory approximation of the exact solution.

We start by the numerical simulation of the complex valued function

$$
f(z)=\log \left(\frac{z-0.2}{z+0.2}\right)
$$

where the canonical form of the complex $z$ is given by $z=x_{1}+i x_{2}, \log$ is the principal determination of the complex Logarithmic function and $f$ is the complex potential of the Rankine irrotational flow around the source $(0.2,0)$ and the sink point $(-0.2,0)$ (see $[6,15])$. The real part $\varphi\left(x_{1}, x_{2}\right)=\Re f(z)$ is the velocity potential and is computed as the solution of a Poisson-Neumann problem set in $\Omega_{\Gamma}^{e}$ (the complementary of the obstacle $\left.\Omega_{\Gamma}^{i}=\right]-0.25,0.25[\times]-0.05,0.05[)$, the imaginary part $\psi\left(x_{1}, x_{2}\right)=\Im f(z)$ represents the stream-function of the flow and satisfies a Poisson-Dirichlet equation. The boundary condition in both cases is written using the integral representation on the obstacle boundary $\Gamma=\partial \Omega_{\Gamma}^{i}$. All two-dimensional computations are realized by linear triangular finite elements.

The first computations on $\Omega_{\Sigma}^{c}$ which is constantly symmetric with respect to both $x_{1}$ - and $x_{2}$-axis are involved in the potential $\varphi$ for different rectangular choices. The fictitious boundary $\Sigma$ is thus perfectly characterized by its length and width $(\ell, w)$. In the left panel of Figure 3, are depicted in a semi-logarithmic scale, the linear regression of the curves, indicating the behavior of the gap (with the discrete $\ell^{2}$-norm) between two consecutive computed potentials $\left(\left\|\varphi^{m+1}-\varphi^{m}\right\|_{\ell^{2}}\right)$ with respect to the iterations number $(m)$. Their structures seem in agreement with the theoretical predictions; indeed a geometrical convergence rate is shown. Furthermore, we observe that the convergence is faster for larger domains of computation.

Now, we investigate the reduced simulations as described in Section 5.2 where we exploit the symmetries of the problem. The function $\varphi$ is oddly and evenly symmetric with respect to the $x_{1}$ - and $x_{2}$-axis. A relevant choice is to achieve the calculations only in a quarter part of the domain $\Omega_{\Sigma}^{c}$. We enforce a Dirichlet condition 

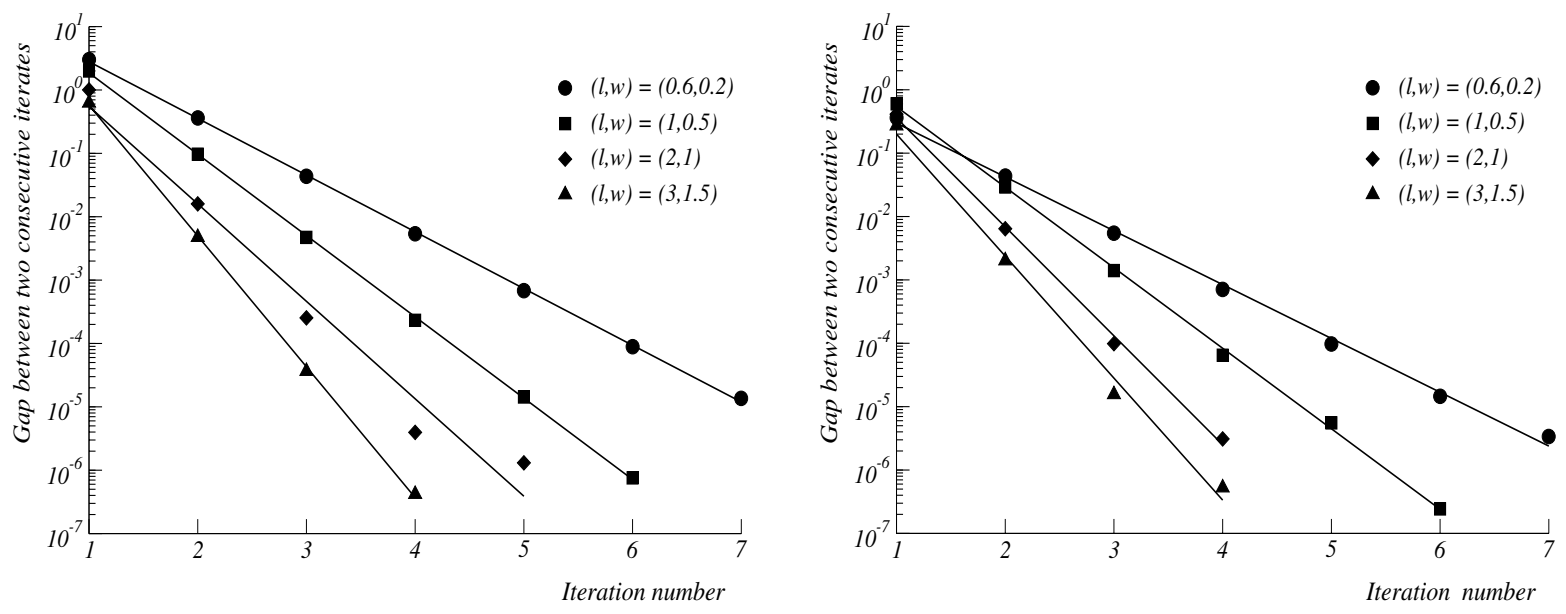

Figure 3. The convergence curves of the potential $\varphi$ (the left panel) and for the streamfunction $\psi$ (the right panel).

TABLE 1. Relative gaps between $(\varphi, \psi)$, the exact solutions and $\left(\varphi^{S c}, \psi^{S c}\right)$ computed by the Schwarz method using both symmetries (corresponding to (NE)-panels in Figs. 4 and 5).

\begin{tabular}{|c|c|c|c|c|}
\hline$(\ell, w)$ & $(0.6,0.2)$ & $(1,0.5)$ & $(2,1)$ & $(3,1.5)$ \\
\hline$\frac{\left\|\varphi-\varphi^{S c}\right\|_{\ell^{2}}}{\|\varphi\|_{\ell^{2}}}$ & 0.0107 & 0.0093 & 0.0081 & 0.0075 \\
\hline$\frac{\left\|\varphi-\varphi^{S c}\right\|_{\ell \infty}}{\|\varphi\|_{\ell^{\infty}}}$ & 0.0168 & 0.0145 & 0.0135 & 0.0131 \\
\hline$\frac{\left\|\psi-\psi^{S c}\right\|_{\ell^{2}}}{\|\psi\|_{\ell^{2}}}$ & 0.0014 & 0.0008 & 0.0003 & 0.0003 \\
\hline$\frac{\left\|\psi-\psi^{S c}\right\|_{\ell \infty}}{\|\psi\|_{\ell^{\infty}}}$ & 0.0060 & 0.0033 & 0.0014 & 0.0017 \\
\hline
\end{tabular}

on the $x_{1}$-axis and a Neumann condition on the $x_{2}$-axis. In Figure 4, are drawn the isolines of $\varphi$ that are obtained by four different computations (when $(\ell, w)=(1,0.5)$ ). The $(\mathrm{SW})^{2}$ panel represents $\varphi$ in the quarter of $\Omega_{\Sigma}^{c}$ located in $\mathbb{R}_{-} \times \mathbb{R}_{-}$, resulting from a simulation in the whole domain. The (SE) panel plots $\varphi$, calculated by using the odd-symmetry, the computations are then realized for the domain $\Omega_{\Sigma}^{c} \cap\left(\mathbb{R} \times \mathbb{R}_{-}\right)$. The (NW) panel depicts $\varphi$ in the quarter of $\Omega_{\Sigma}^{c}$ located in $\mathbb{R}_{-} \times \mathbb{R}_{+}$, provided by a simulation exploiting the even-symmetry. The final (NE) panel is obtained by calculations that take into account both symmetries. The regularity of the isolines distribution and the apparently perfect even and odd symmetries, readily confirmed by the evaluation of some relevant indicators reported in Table 1 and are encouraging to carry out reduced computations whenever it is possible.

The simulation of the stream-function $\psi$ is obtained by solving the Poisson-Dirichlet equation in $\Omega_{\Gamma}^{e}$ keeping unchanged the meshes used in the previous calculations. The observations that can be drawn from the Figures 3 and 5 are very similar to those made for the Neumann condition on the obstacle. In fact, the error curves depicted in the right diagram of Figure 3 illustrates a geometrical convergence of the Schwarz method. Moreover, Figure 5 and the two last rows of Table 1 show that the use of the symmetries to solve reduced problems benefits to the computational complexity.

\footnotetext{
${ }^{2}$ For South-West panel.
} 

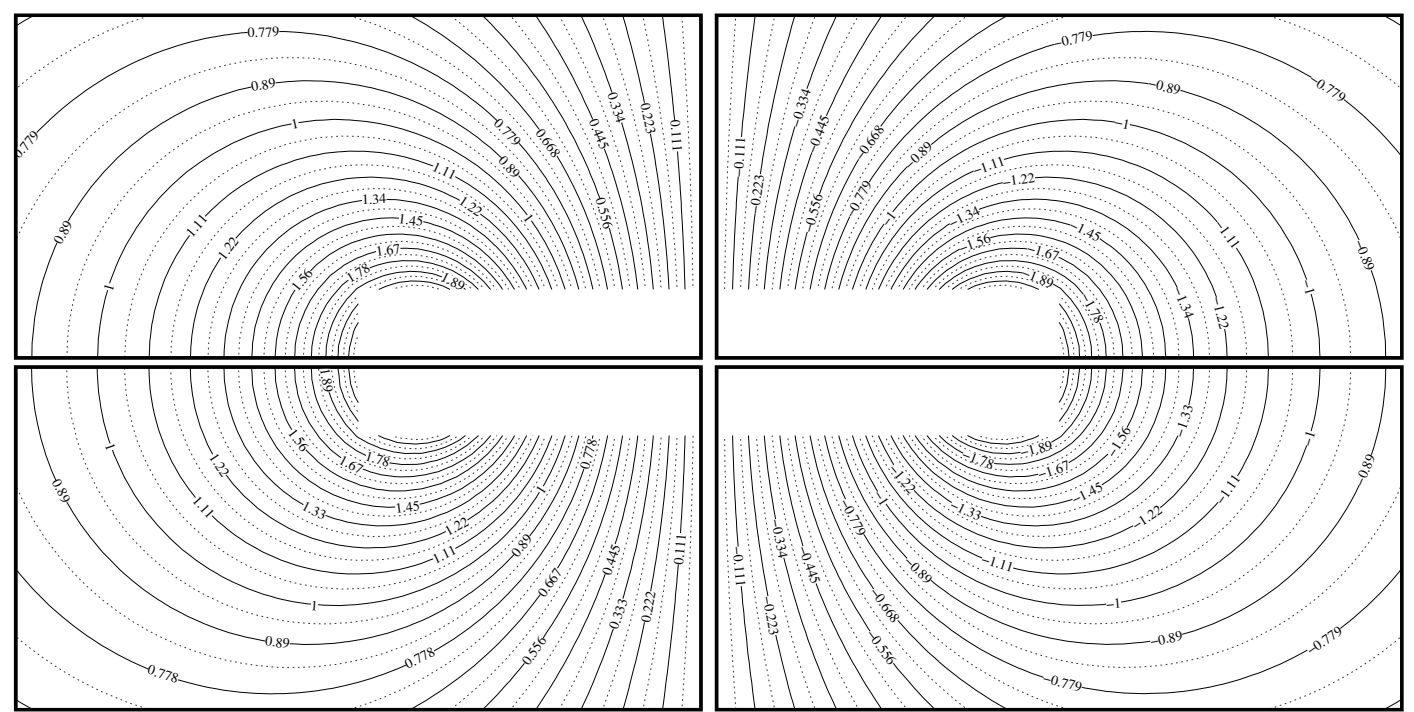

FIGURE 4. Isolines of the Rankine potential function $\varphi$. Each quarter is simulated by a different approach due to the $x_{1}$-odd and $x_{2}$-even symmetries. For the panel (NE) both symmetries are used, for the panel (NW) the $x_{2}$-symmetry is used, for the panel (SW) none of the symmetries is used and for the panel (SE) the $x_{1}$-symmetry is used.
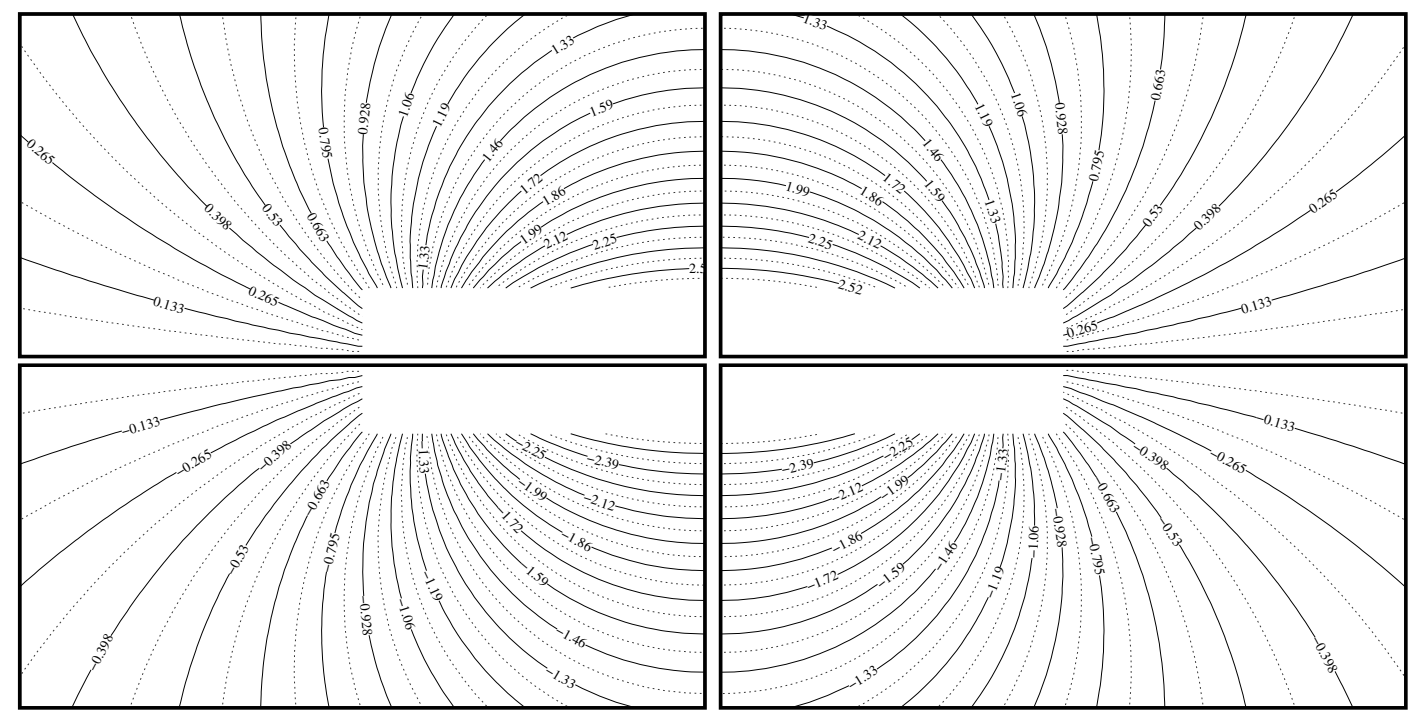

FIGURE 5. Isolines of the Rankine stream-function $\psi$ obtained by reduced computations. The symmetries of $\psi$ are dual to those of $\varphi$ (see Fig. 4).

For an additional checking of the Schwarz approach, we conduct two elementary numerical experiments for the three-dimensional Poisson-Neumann problem. Our ultimate goal is to put emphasize on the geometrical convergence of it. 


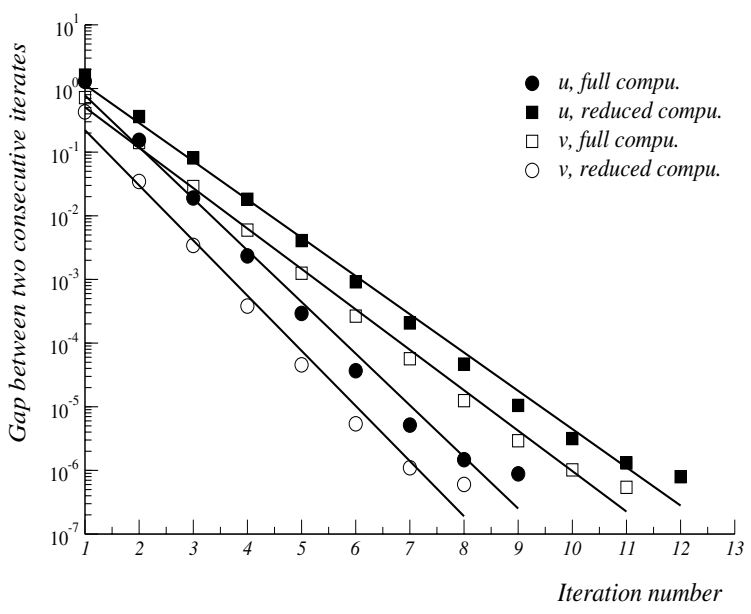

FiguRE 6. The Schwarz convergence curves for $u^{S c}$ and $v^{S c}$ in three-dimensions. Reduced computations are achieved in the quarter of the spheroid owing to the symmetries.

TABLE 2. Relative errors $\left(u-u^{S c}\right)$ and $\left(v^{C o}-v^{S c}\right)$ with respect to the $\ell^{2}$ - and $\ell^{\infty}$-norms.

\begin{tabular}{|c|c|c|c|c|}
\hline & $\frac{\left\|u-u^{S c}\right\|_{\ell^{2}}}{\|u\|_{\ell^{2}}}$ & $\frac{\left\|u-u^{S c}\right\|_{\ell \infty}}{\|u\|_{\ell \infty}}$ & $\frac{\left\|v^{C o}-v^{S c}\right\|_{\ell^{2}}}{\left\|v^{C o}\right\|_{\ell^{2}}}$ & $\frac{\left\|v^{C o}-v^{S c}\right\|_{\ell \infty}}{\left\|v^{C o}\right\|_{\ell \infty}}$ \\
\hline Full computations & 0.0024 & 0.0092 & $0.45 \times 10^{-6}$ & $0.14 \times 10^{-5}$ \\
\hline Reduced computations & 0.0028 & 0.0071 & $0.52 \times 10^{-6}$ & $0.18 \times 10^{-5}$ \\
\hline
\end{tabular}

One corresponds to the exact solution

$$
u(\boldsymbol{x})=u\left(x_{1}, x_{2}, x_{3}\right)=\frac{1}{\sqrt{\left(x_{1}-0.5\right)^{2}+x_{2}^{2}+x_{3}^{2}}},
$$

and the solution of the other, denoted by $v$, is specified by the Neumann condition

$$
\frac{\partial v}{\partial n}=\left(\cos \left(2 \pi\left(x_{1}-0.5\right)\right), \sin \left(2 \pi x_{2}\right),-x_{3}\right) \cdot n .
$$

Notice that we have not a closed form of $v$; we then consider $v^{c o}$ as the reference solution to which $v^{S c}$ is to be compared. The exterior domain is the complementary part of the unit ball. The fictitious Dirichlet condition is applied on the sphere centered at the origin and of radius 1.2. The computational domain is then the spheroid of double radii 1 and 1.2. Moreover, in both cases, the even symmetries with respect to the plans $x_{2}=0$ and $x_{3}=0$ may be taken into account to run the calculations only in a quarter of the spheroid (the one located in the quarter-space $x_{2} \geq 0$ and $\left.x_{3} \leq 0\right)$. The approximated solutions $u^{S c}, v^{S c}$ and $v^{C o}$ are obtained by using quadratic prismatic finite elements. In Figure 6 are plotted, for both tests, the convergence historic for reduced and full computations. Table 2 displays the accuracy of the solutions $u^{S c}$ and $v^{S c}$ compared to $u$ and $v^{C o}$ respectively. The curves illustrate again the geometrical convergence rate of the Schwarz approach in both examples and can be exempted from any further comments. 


\section{Conclusion}

The adaptation made on the original Schwarz method, to fit the particular features of the exterior problems, does improve its capabilities. It enables us to resort to suitable integral representations for solving the subproblems containing the condition at infinity. The analysis and the numerical discussion in this contribution increase the efficiency of the Schwarz approach while saving valuable computational time. The main advantage of the new method is that any scientific program, dealing with boundary value problems in bounded domains, can be transformed to take into account exterior problems, by simply adding some elementary procedures of regular integral representations. The remaining challenge is to assess the ability of this tool to approximate more relevant models, such as the eddy current equation or the Maxwell system. For further details in many formulations of the (Schwarz) algorithm and numerical discussion in the case of the electro-technical problems, we refer to F. Jelassi's Ph.D. thesis [24].

Acknowledgements. We are indebted to Dr D. Martin, from the Université de Rennes, to whom we address our special thanks, for his valuable help and for providing us with his Computing Code MELINA.

\section{REFERENCES}

[1] R.A. Adams, Sobolev Spaces. Academic Press (1975).

[2] C. Albuquerque and G.-H. Cottet, Coupling finite difference methods and integral formulas for elliptic problems arising in fluid mechanics. Numer. Methods Partial Differential Equations 20 (2003) 199-229.

[3] F. Ben Belgacem, M. Fournié, N. Gmati and F. Jelassi, Sur le traitement des conditions aux limites à l'infini pour quelques problèmes extérieurs par la méthode de Schwarz alternée. (French) [Handling boundary conditions at infinity for some exterior problems by the alternating Schwarz method] C. R. Math. Acad. Sci. Paris 336 (2003) 277-282.

[4] P.E. Bjørstad, Multiplicative and additive Schwarz methods: Convergence in the two domain case, in Second International Domain Decomposition Methods for Partial Differential Equations, T.F. Chan, R. Glowinski, J. Périaux and O.B. Widlund Eds., SIAM, Philadelphia (1989) 147-159.

[5] M. Bonnet, Equations intégrales et éléments de frontière. CNRS, Éditions Eyrolles, Paris (1995).

[6] P. Chassaing, Mécanique des fluides. Eléments d'un premier parcours. Collection Polytech, Cépaduès-Editions, $2^{\mathrm{e}}$ édition (2000).

[7] S. Christiansen and J.C. Nédélec, A preconditioner for the Electric Field Integral Equation based on Calderon formula. SIAM J. Numer. Anal. 40 (2002) 1100-1135.

[8] D.L. Colton and R. Kress, Integral equation methods in scattering theory. Pure Appl. Math., Wiley-Interscience, John Wiley \& Sons, New York (1983).

[9] G.-H. Cottet, Particle grid domain decomposition methods for the Navier-Stokes equations in exterior domains. C. Anderson and C. Greengard Eds., Vortex dynamics and vortex methods, American Mathematical Society, Rhode Island (1991) $103-117$.

[10] R. Dautray and J.-L.Lions, Analyse mathématique et calcul numérique pour les sciences et les techniques, Collaboration avec M. Artola, P. Bénilan, M. Bernadou, M. Cessenat, J.-C. Nédélec et J. Planchard. Réimprimé à partir de l'édition de 1984. INSTN: Collection Enseignement, Masson, Paris (1988).

[11] M. Dryja and O.B. Widlund, Some domain decomposition algorithms for elliptic problems, in iterative Methods for Large Linear Systems. L. Hayes and D. Kincaid Eds., Academic Press, San Diego, CA (1989).

[12] M. Dryja and O.B. Widlund, Towards a unified theory of domain decomposition algorithms for elliptic problems, in Third International Symposium on Domain Decomposition Methods for Partial Differential Equations, T.F. Chan, R. Glowinski, J. Périaux, O.B. Widlund, Eds., SIAM, Philadelphia (1990) 273-291.

[13] D. Euvrard, Résolution numérique des équations aux dérivées partielles. Masson, Paris (1990).

[14] M. Feistauer and C. Schwab, Coupled Problems for Viscous Incompressible Flow in Exterior Domains. A. Sequeira et al. Eds., Kluwer/Plenum Publ. Appl. Nonlinear Anal. (1999) 97-116.

[15] P. Germain and P. Muller, Introduction à la mécanique des milieux continus. 2e édition, Masson, Paris (1990).

[16] J. Giroire, Études de quelques problèmes aux limites extérieurs et résolution par équations intégrales. Thèse de Doctorat d'État, Université Pierre et Marie Curie, Paris VI (1987).

[17] J. Giroire and J.-C. Nédélec, Numerical solution of an exterior Neumann problem using a double-layer potential. Math. Comp. 32 (1978) 973-990.

[18] L. Greengard and V. Rokhlin, A new version of the fast multipole method for the Laplace equation in 3 dimensions. Cambridge University Press, Cambridge, UK. Acta Numerica 6 (1997) 226-269.

[19] J.D. Jackson, Classical electrodynamics. Wiley, New York, third edition (1999). 
[20] A. Jami, Résolution numérique des problèmes de Helmholtz extérieurs par couplage entre éléments finis et représentation intégrale. C. R. Acad. Sci. Paris Sér. A-B 287 (1978) A799-A801.

[21] A. Jami and M. Lenoir, Formulation variationnelle pour le couplage entre une méthode d'éléments finis et une représentation intégrale. C. R. Acad. Sci. Paris Sér. A-B 285 (1977) A269-A272.

[22] A. Jami and M. Lenoir, A new numerical method for solving exterior linear elliptic problems. Sixth International Conference on Numerical Methods in Fluid Dynamics (Proc. Conf., Tbilisi, 1978), Springer, Berlin-New York. Lect. Notes Phys. 90 (1979) $292-298$.

[23] M.A. Jawson and G.T. Symm, Integral Equations Methods in Potential Theory and Elastostatics. Academic Press, New York (1977).

[24] F. Jelassi, Ph.D. Thesis of the University Paul Sabatier Toulouse (France) and the École Nationale d'Ingénieurs de Tunis (Tunisia).

[25] C. Johnson and J.-C. Nédélec, On the coupling of boundary integral and finite element methods. Math. Comput. 35 (1980) 1063-1079.

[26] C. Hazard and M. Lenoir, Modélisation et résolution des problèmes de diffraction. Cours de L'ENSTA et de DEA de Mécanique, Paris VI, ENSTA SMP, Centre de l'Yvette, Palaiseau (1995).

[27] M.-N. Le Roux, Résolution numérique du problème du potentiel dans le plan par une méthode variationnelle d'éléments finis. Thèse de Doctorat de $3^{\mathrm{e}}$ cycle, Université de Rennes (1974).

[28] M.-N. Le Roux, Méthode d'éléments finis Résolution pour la résolution des problèmes extérieurs en dimension deux. RAIRO Anal. Numér. 11 (1977) 27-60.

[29] M. Lenoir, Equations intégrales et problèmes de diffraction. Cours de L'ENSTA, Paris VI, ENSTA SMP, Centre de l'Yvette, Palaiseau (2003).

[30] P.-L. Lions, On the alternating Schwarz method I, in First International Symposium on Domain Decomposition Methods for Partial Differential Equations. R. Glowinski, G. H. Golub, G. A. Meurant and J. Périaux, Eds., SIAM, Philadelphia (1988) $1-42$.

[31] P.-L. Lions, On the alternating Schwarz method II, in Second International Domain Decomposition Methods for Partial Differential Equations. T.F. Chan, R. Glowinski, J. Périaux and O.B. Widlund, Eds., SIAM, Philadelphia, (1989) 47-70.

[32] J. Liu and J.-M. Jin, A novel hybridization of higher order finite element and boundary integral methods for electromagnetic scattering and radiation problems. IEEE Trans. Antennas Propagation 49 (2001) 1794-1806.

[33] J. Liu and J.-M. Jin, A Highly effective preconditioner for solving the finite element-boundary integral matrix equation of 3-D scattering. IEEE Trans. Antennas and Propagation 50 (2002) 1212-1221.

[34] D. Martin, MELINA, Guide de l'utilisateur. IRMAR, Université de Rennes I et ENSTA Paris (2000). http://perso.univ-rennes1.fr/daniel.martin/melina/

[35] A.M. Matsokin and S.V. Nepomnyaschikh, A Schwarz alternating method in a subspace. Soviet Math. 29 (1985) $78-84$.

[36] J.-C. Nédélec, Approximation des équations intégrales en mécanique et en physique. Cours de DEA, Centre de mathématiques appliquées-école polytechnique (1977).

[37] J.-C. Nédélec, Acoustic and Electromagnetic equations. Integral Representations for Harmonic Problems. Springer-Verlag, New-York, Appl. Math. Sci. 144 (2001).

[38] A. Quarteroni and R. Valli, Domain Decomposition Methods for Partial Differential Equations, Numerical mathematics and Scientific computation. Oxford Science Publications (1999).

[39] H.A. Schwarz, Gesammelte Mathematische Abhandlungen, Volume 2. Springer, Berlin (1890). First published in Vierteljahrsschrift Naturforsch. Ges. Zurich (1870).

[40] A. Sequeira, Couplage de la méthode des éléments finis et des équations intégrales - Application au problème de Stokes stationnaire dans le plan. Thèse de Doctorat de $3^{\mathrm{e}}$ cycle, Université Paris 6 (1981).

[41] A. Sequeira, The Coupling of boundary integral and finite element methods for the bi-dimensional exterior steady Stokes problem. Math. Methods Appl. Sci. 5 (1983) 356-375.

[42] P. Silvester and M.S. Hsieh, Finite element solution for two-dimensional exterior field problem. Proc. Inst. Electr. Eng. 118 (1971) 1743-1746.

[43] B. Smith, P. Bjørstad and W. Gropp, Domain Decomposition Method Parallel Multilevel Methods for Elliptic Partial Differential Equations. Cambridge university press, Cambridge (1996).

[44] I. Stakgold, Green functions and boundary value problems. Pure Appl. Math., Wiley-Interscience, John Wiley \& Sons, New York, Second edition (1998).

[45] O. Steinbach and W.L. Wendland, The construction of some efficient preconditioners in the boundary element method. Adv. Comput. Math. 9 (1998) 191-216.

[46] W.L. Wendland, Boundary element methods for elliptic problems, in Mathematical Theory of Finite Element and Boundary Element Methods. A.H. Schatz, V. Thomée, W.L. Wendland Eds., Birkhäuser Verlag, Bazsel (1990).

[47] O. Zienkiewicz, D.W. kelly and P. Bettess, The coupling of the finite element method and boundary solution procedures. Internat. J. Numer. Methods Engrg. 11 (1977) 355-375. 